\title{
User Scheduling for Millimeter Wave Hybrid Beamforming Systems with Low-Resolution ADCs
}

\author{
Jinseok Choi, Gilwon Lee, and Brian L. Evans
}

\begin{abstract}
We investigate uplink user scheduling for millimeter wave (mmWave) hybrid analog/digital beamforming systems with low-resolution analog-to-digital converters (ADCs). Deriving new scheduling criteria for the mmWave systems, we show that the channel structure in the beamspace, in addition to the channel magnitude and orthogonality, plays a key role in maximizing the achievable rates of scheduled users due to quantization error. The criteria show that to maximize the achievable rate for a given channel gain, the channels of the scheduled users need to have (1) as many propagation paths as possible with unique angle-of-arrivals (AoAs) and (2) even power distribution in the beamspace. Leveraging the derived criteria, we propose an efficient scheduling algorithm for mmWave zero-forcing receivers with low-resolution ADCs. We further propose a chordal distance-based scheduling algorithm that exploits only the AoA knowledge and analyze the performance by deriving ergodic rates in closed form. Based on the derived rates, we show that the beamspace channel leakage resulting from phase offsets between AoAs and quantized angles of analog combiners can lead to sum rate gain by reducing quantization error compared to the channel without leakage. Simulation results validate the sum rate performance of the proposed algorithms and derived ergodic rate expressions.
\end{abstract}

\section{Index Terms}

Millimeter wave, low-resolution ADCs, hybrid MIMO system, user scheduling, channel structure.

\section{INTRODUCTION}

Millimeter wave wireless communication has emerged as a promising technology for next generation cellular systems [1]. The advantages of remarkably wide bandwidth in mmWave

J. Choi and B. L. Evans are with the Wireless Networking and Communication Group (WNCG), Dept. of Electrical and Computer Engineering, The University of Texas at Austin, Austin, TX 78701. (e-mail: \{jinseokchoi89@, bevans@ece.\}utexas.edu). G. Lee is with Intel Corporation, Santa Clara, CA 95054. (e-mail: gilwon.lee@ intel.com). J. Choi and B. L. Evans were supported by gift funding from Huawei Technologies. A preliminary version of this work was presented in IEEE ICC 2018 [4]. 
frequencies ranging from $30-300 \mathrm{GHz}$ can be exploited to meet ever increasing capacity requirements of wireless communication network. To compensate for the large path loss of mmWave channels, large antenna arrays are likely to be deployed into tranceivers with very small antenna spacing owing to the small wavelength. Due to a large signal bandwidth, highresolution ADCs coupled with large antenna arrays demand significant power consumption in the receiver, and the power consumption of ADCs scales exponentially in the number of quantization bits. Therefore, employing low-resolution ADCs has been proposed as a natural solution, and extensive research has been conducted in such systems for mmWave communications [5], [6]. In this regard, as an extension of our work [7], we also investigate low-resolution ADC systems by focusing on user scheduling.

\section{A. Prior Work}

As an effort to realize low-resolution ADC systems, essential wireless communication techniques such as channel estimation and detection have been developed in low-resolution ADC systems [8]-[10], [12]-[14]. For the 1-bit ADC system which is the extreme case of low-resolution ADCs, compressive sensing [8], maximum-likelihood [9], and Bussgang decomposition-based techniques [10] were employed for channel estimation. Compressive sensing-based channel estimators were also developed for the systems with low-resolution ADCs [12], and achieved comparable estimation accuracy to that of infinite-bit ADC systems at low and medium signalto-noise ratio (SNR). Achieving higher detection accuracy than a minimum mean squared error (MMSE) estimator, message passing de-quantization-based detectors were proposed in 1-bit ADC [13] and low-resolution ADC systems [14].

In recent years, low-resolution ADC systems with hybrid analog/digital beamforming have been investigated to take advantage of both the reduced number of ADC bits and radio frequency (RF) chains [15]-[18]. It was shown in [15] that the hybrid beamforming systems with lowresolution ADCs achieve comparable rate to that of infinite-bit ADC systems, providing better energy-rate trade-off compared to conventional hybrid multiple-input multiple-output (MIMO) systems and low-resolution ADC systems. To further increase spectral and energy efficiency of mmWave receivers, deploying adaptive-resolution ADCs in hybrid MIMO systems was proposed with ADC bit-allocation algorithms [16], [17]. Channel estimation techniques were also investigated for hybrid MIMO systems with low-resolution ADCs [18]. Understanding the superior 
spectral and energy efficiency of the architecture, we focus on the hybrid MIMO receiver with low-resolution ADCs to solve a user scheduling problem in mmWave communications.

Although user scheduling in multiuser MIMO systems has been extensively studied for more than a decade, it has not been investigated for low-resolution ADC systems. One representative method of user scheduling is the semi-orthogonal user selection (SUS) method [19]. This method selects users in a greedy manner such that the channel vectors of the selected users are nearly orthogonal and have large magnitudes based on the full channel state information (CSI) knowledge of all users at the basestation (BS). Another representative approach is the random beamforming (RBF) method [20] that selects the user who has the maximum signal-tointerference-noise ratio (SINR) for each beam when a set of orthogonal beams are determined a priori at the BS before scheduling. Similarly, to capture the orthogonality between channels of scheduled users, user scheduling algorithms that adopt chordal distance as a selection measure were proposed in [21], [22].

Unlike the user scheduling methods that have been studied under the Rayleigh fading channel model by assuming rich scattering [19], [20], [24], different approaches have investigated user scheduling under the channels with poor scattering such as mmWave channels [26]-[28]. In [26], user scheduling algorithms were proposed for mmWave communications by leveraging the knowledge of channel gain and angle of departure. In addition, the achievable sum rate was quantified for the BS which employs an iterative matrix decomposition based hybrid beamforming scheme proposed in [29]. The RBF method was analyzed in both the uniform random single path [27] and multi-path channel models [28]. By exploiting the sparse nature of mmWave channels, beam aggregation-based scheduling and fairness-aware scheduling algorithms were developed in [28]. Although the user scheduling algorithms were proposed for mmWave communications, they still focused on user scheduling without quantization error. Consequently, user scheduling in mmWave systems with low-resolution ADCs remains questionable.

\section{B. Contributions}

In this paper, we investigate uplink user scheduling for mmWave hybrid MIMO zero-forcing receivers with low-resolution ADCs. Noting that non-negligible quantization error can be a primary bottleneck for attaining scheduling gain in the low-resolution ADC system, we provide following contributions: 
- We derive user scheduling criteria to maximize the scheduling gain by finding the best tradeoff between channel gains and corresponding quantization noise. Adopting the virtual channel model [30], the criteria can be interpreted as follows: for a given channel gain, $(i)$ unique AoAs of each scheduled user and $(i i)$ equal power spread across the beamspace complex gains within each user maximize sum rate. Accordingly, the derived scheduling criteria reveal that the channel structure in the beamspace, in addition to the channel magnitude and orthogonality, plays a key role in maximizing sum rate under coarse quantization.

- Leveraging the derived criteria, we propose an efficient scheduling algorithm for hybrid lowresolution ADC systems. The proposed algorithm combines semi-orthogonal user filtering [19] and non-overlap filtering of dominant beams [28] to enforce orthogonality among scheduled users and to reduce quantization error. Using an approximated SINR as a scheduling measure, the algorithm captures the trade-off between channel gain and corresponding quantization error, and reduces computational complexity by avoiding matrix inversion.

- Considering the difficulty of acquiring instantaneous full CSI, we further propose a chordal distance-based scheduling algorithm which only requires AoAs of mmWave channels, known as slowly-varying channel characteristics [31]. Unlike the previously developed chordal distancebased algorithms [21], [22] that use full CSI and adopt a simple greedy structure which requires prohibitively high complexity, our proposed algorithm exploits only the AoA information of mmWave channels and reduces the complexity by filtering a user candidate set.

- To analyze the performance of the chordal distance-based algorithm, we derive closed-form sum rates for two channel scenarios: (1) AoAs exactly align with quantized angles of analog combiners and (2) arbitrary AoAs produce phase offsets from the quantized angles, which results in channel leakage. For the first scenario, we derive an ergodic rate as the sum of the ergodic rate with no quantization and the rate loss due to quantization. Accordingly, the derived rate provides the expected ergodic rate loss due to quantization in closed form. For the second scenario, an approximated lower bound of the ergodic rate is derived in closed form. We observe that the two channel scenarios result in different sum rates as a consequence of coarse quantization, and the channel leakage provides sum rate gain by reducing quantization error, which challenges the conventional negative understanding towards channel leakage.

Simulation results demonstrate the superior ergodic sum rate performance of the proposed algorithms and validate the analysis and intuition obtained in this paper.

Notation: $\mathbf{A}$ is a matrix and $\mathbf{a}$ is a column vector. $\mathbf{A}^{H}$ and $\mathbf{A}^{\top}$ denote conjugate transpose 


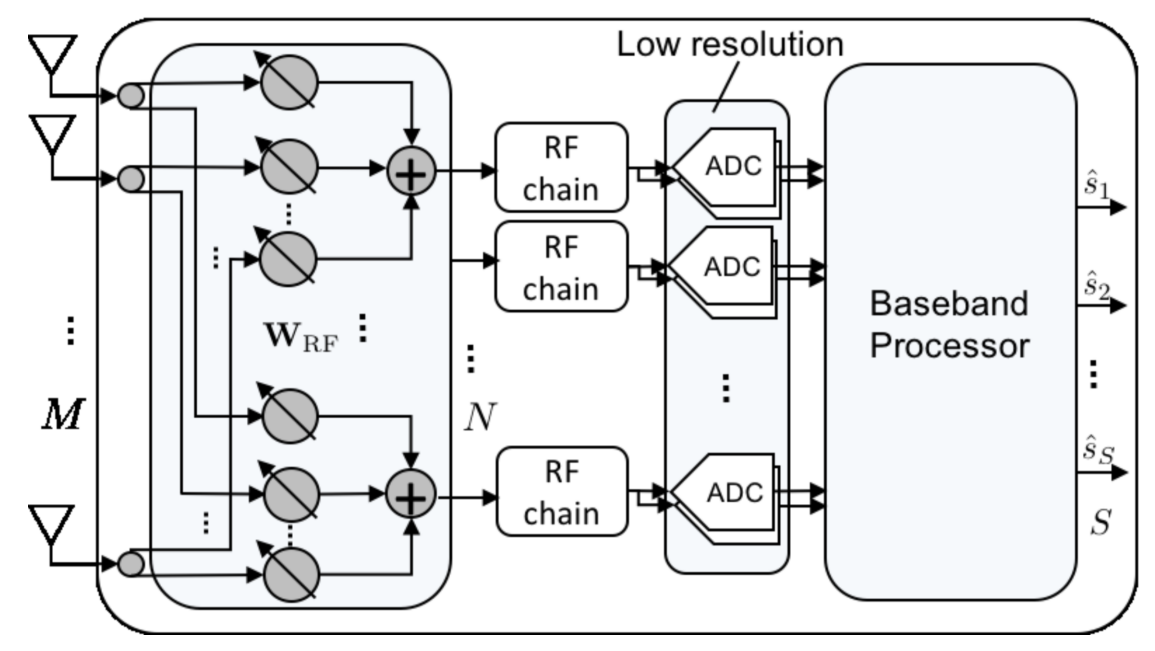

Fig. 1. A receiver architecture with large antenna arrays and analog combiners $\mathbf{W}_{\mathrm{RF}}$, followed by low-resolution ADCs.

and transpose. $[\mathbf{A}]_{i,:}$ and $\mathbf{a}_{i}$ indicate the $i$ th row and column vector of $\mathbf{A}$. We denote $a_{i, j}$ as the $\{i, j\}$ th element of $\mathbf{A}$ and $a_{i}$ as the $i$ th element of $\mathbf{a} \cdot \mathcal{C N}\left(\mu, \sigma^{2}\right)$ is a complex Gaussian distribution with mean $\mu$ and variance $\sigma^{2} . \mathbb{E}[\cdot]$ and $\operatorname{Var}[\cdot]$ represent expectation and variance operator, respectively. The cross-correlation matrix is denoted as $\mathbf{R}_{\mathbf{x y}}=\mathbb{E}\left[\mathbf{x y}^{H}\right]$. The diagonal matrix $\operatorname{diag}(\mathbf{A})$ has $\left\{a_{i, i}\right\}$ at its $i$ th diagonal entry, and $\operatorname{diag}(\mathbf{a})$ or $\operatorname{diag}\left(\mathbf{a}^{\top}\right)$ has $\left\{a_{i}\right\}$ at its $i$ th diagonal entry. $\mathbf{I}_{N}$ denotes an $N \times N$ identity matrix and $\|\mathbf{A}\|$ represents $L_{2}$ norm. $|\cdot|$ indicates an absolute value for a complex value or denotes cardinality of a set. $\operatorname{tr}(\cdot)$ is a trace operator.

\section{SySTEM MODEL}

\section{A. Signal and Channel Models}

We consider a single-cell multiuser MIMO network for uplink communications. A BS employs a uniform linear array (ULA) of $M$ receive antennas. Analog combiners are applied at the BS, followed by $N \leq M$ chains as shown in Fig. 1. We assume that $K$ single-antenna users are distributed in the cell and the BS schedules $S \leq N$ users to serve among the $K$ users in the cell. The ADCs are considered to be low-resolution ADCs to reduce the receiver power consumption.

Focusing on mmWave communications, the channel $\mathbf{h}_{k}$ for user $k$ is assumed to be a sum of the contributions of limited scatterers that contribute $L_{k}$ propagation paths to the channel $\mathbf{h}_{k}$ [32]. Therefore, the discrete-time narrowband channel of user $k$ can be modeled as [30]

$$
\mathbf{h}_{\gamma, k}=\sqrt{\frac{1}{\gamma_{k}}} \mathbf{h}_{k}=\sqrt{\frac{M}{\gamma_{k} L_{k}}} \sum_{\ell=1}^{L_{k}} g_{k, \ell} \mathbf{a}\left(\phi_{k, \ell}\right)
$$


where $\gamma_{k}$ denotes the pathloss of user $k, g_{k, \ell}$ is the complex gain of the $\ell$ th propagation path of user $k$, and $\mathbf{a}\left(\phi_{k, \ell}\right)$ is the array steering vector of the $\mathrm{BS}$ receive antennas corresponding to the azimuth AoA of the $\ell$ th path of the $k$ th user $\phi_{k, \ell} \in[-\pi / 2, \pi / 2]$. We consider that $g_{k, \ell}$ is an independent and identically distributed (IID) complex Gaussian random variable as $g_{k, \ell} \stackrel{i . i . d}{\sim} \mathcal{C N}(0,1)$. The array steering vector $\mathbf{a}(\theta)$ for the ULA antennas of the BS is given as

$$
\mathbf{a}(\theta)=\frac{1}{\sqrt{M}}\left[1, e^{-j \pi \vartheta}, e^{-j 2 \pi \vartheta}, \ldots, e^{-j(M-1) \pi \vartheta}\right]^{\top}
$$

where $\vartheta=\frac{2 d}{\lambda} \sin (\theta)$ is the spatial angle that is related to the physical AoA $\theta, d$ denotes the distance between antenna elements, and $\lambda$ represents the signal wave length. Throughout this paper, we use $\theta$ and $\phi$ to denote the physical angles of analog combiners and physical AoAs of a user channel, respectively. We also use $\vartheta$ and $\varphi$ to indicate the spatial angles for $\theta$ and $\phi$, respectively. We assume that $\vartheta$ is a constant value in the range of $[-1,1]$ and $\varphi$ is a uniform random variable $\varphi \sim \operatorname{Unif}[-1,1]$.

For simplicity, we consider a homogeneous long-term received SNR network ${ }^{1}$ where a conventional uplink power control compensates for the pathloss and shadowing effect to achieve the same long-term received SNR target for all users in the cell [33], [34]. Let $\mathbf{x}=\mathbf{P s}$ be the transmitted user signals where $\mathbf{P}=\operatorname{diag}\left\{\sqrt{\rho \gamma_{1}}, \ldots, \sqrt{\rho \gamma_{S}}\right\}$ is the transmit power matrix and $\mathbf{s}$ is the $S \times 1$ transmitted symbol vector from $S$ users. Let $\mathbf{H}_{\gamma}=\mathbf{H B}$ represent the $M \times S$ channel matrix where $\mathbf{H}_{\gamma}=\left[\mathbf{h}_{\gamma, 1}, \ldots, \mathbf{h}_{\gamma, S}\right]$ is the channel matrix, $\mathbf{H}=\left[\mathbf{h}_{1}, \ldots, \mathbf{h}_{S}\right]$ is the channel matrix after the uplink power control, and $\mathbf{B}=\operatorname{diag}\left\{\sqrt{1 / \gamma_{1}}, \ldots, \sqrt{1 / \gamma_{S}}\right\}$ is the pathloss matrix. Then, the received baseband analog signal $\mathbf{r} \in \mathbb{C}^{M}$ is given as

$$
\mathbf{r}=\mathbf{H}_{\gamma} \mathbf{x}+\mathbf{n}=\mathbf{H B P} \mathbf{s}+\mathbf{n}=\sqrt{\rho} \mathbf{H} \mathbf{s}+\mathbf{n}
$$

where we assume $\mathbf{s} \sim \mathcal{C N}\left(\mathbf{0}, \mathbf{I}_{S}\right)$, and $\mathbf{n}$ indicates the additive white Gaussian noise (AWGN) vector $\mathbf{n} \sim \mathcal{C N}\left(\mathbf{0}, \mathbf{I}_{M}\right)$. Thus, we can regard $\rho$ as the $\mathrm{SNR}$.

The received analog signals in (3) are combined via an $M \times N$ analog combiner $\mathbf{W}_{\mathrm{RF}}$. The combiner $\mathbf{W}_{\mathrm{RF}}$ is implemented using analog phase shifters, and its elements are constrained to have the equal norm of $1 / \sqrt{M}$. After analog combining, (3) becomes

$$
\mathbf{y}=\mathbf{W}_{\mathrm{RF}}^{H} \mathbf{r}=\sqrt{\rho} \mathbf{W}_{\mathrm{RF}}^{H} \mathbf{H} \mathbf{s}+\mathbf{W}_{\mathrm{RF}}^{H} \mathbf{n} .
$$

\footnotetext{
${ }^{1}$ We remark that the proposed scheduling criteria and the proposed algorithms in this paper can also be applicable to a heterogeneous long-term received SNR network.
} 
Assuming uniformly-spaced spatial angles, the matrix of array steering vectors $\mathbf{A}=\left[\mathbf{a}\left(\theta_{1}\right), \ldots\right.$, $\left.\mathbf{a}\left(\theta_{M}\right)\right]$ becomes a unitary discrete Fourier transform (DFT) matrix. Noting that the antenna space and beamspace are related through a spatial Fourier transform, we adopt a sub-matrix of the DFT matrix as the analog combiner $\mathbf{W}_{\mathrm{RF}}=\tilde{\mathbf{A}}$ [16], [35] to project the received signals onto the beamspace, where $\tilde{\mathbf{A}}$ consists of $N$ columns of $\mathbf{A}$. Through the projection, the BS can exploit the sparsity of the mmWave channels to capture channel gains with the reduced number of $\mathrm{RF}$ chains [36]. Using $\mathbf{W}_{\mathrm{RF}}=\tilde{\mathbf{A}}$, we rewrite (4) as

$$
\mathbf{y}=\sqrt{\rho} \tilde{\mathbf{A}}^{H} \mathbf{H} \mathbf{s}+\tilde{\mathbf{A}}^{H} \mathbf{n}=\sqrt{\rho} \mathbf{H}_{\mathrm{b}} \mathbf{s}+\mathbf{v} .
$$

We denote $\mathbf{H}_{\mathrm{b}}=\tilde{\mathbf{A}}^{H} \mathbf{H}$, which is the projection of the channel matrix onto the beamspace. Since $\mathbf{A}$ is a unitary matrix, the projected noise vector $\mathbf{v}=\tilde{\mathbf{A}}^{H} \mathbf{n}$ is distributed as $\mathcal{C N}\left(\mathbf{0}, \mathbf{I}_{N}\right)$.

\section{B. Quantization Model}

In this subsection, we introduce an additive quantization noise model [37] which approximates quantization process in a linear form for analytical tractability. Such linear approximation of quantization provides reasonable accuracy in low and medium SNR ranges [5]. After processed through the RF chains, each complex sample $y_{i}$ in (5) is quantized at the $i$ th pair of ADCs, and each ADC quantizes either a real or imaginary component of $y_{i}$. The quantized signal $\mathbf{y}_{\mathrm{q}}$ is [37]

$$
\mathbf{y}_{\mathrm{q}}=\mathcal{Q}(\operatorname{Re}\{\mathbf{y}\})+j \mathcal{Q}(\operatorname{Im}\{\mathbf{y}\})=\alpha \sqrt{\rho} \mathbf{H}_{\mathrm{b}} \mathbf{s}+\alpha \mathbf{v}+\mathbf{q}
$$

where $\mathcal{Q}(\cdot)$ is the element-wise quantizer function. The quantization gain $\alpha$ is defined as $\alpha=$ $1-\beta, \beta=\mathbb{E}\left[\left|y-y_{\mathrm{q}}\right|^{2}\right] / \mathbb{E}\left[|y|^{2}\right]$ is a normalized mean squared quantization error, and $\mathbf{q}$ is the additive quantization noise vector.

For a scalar MMSE quantizer of a Gaussian random variable, $\beta$ can be approximated as $\beta \approx \frac{\pi \sqrt{3}}{2} 2^{-2 b}$ for $b>5[38]$ where $b$ denotes the number of quantization bits for each real and imaginary part of $y$. The values of $\beta$ for $b \leq 5$ are shown in Table 1 in [16]. Although the quantization error is neither Gaussian nor is its covariance matrix diagonal in an exact nonlinear quantization model, we provide approximations based on [5], [37], [39] as follows: considering a lower bound of achievable rate, we assume $\mathbf{q} \sim \mathcal{C N}\left(\mathbf{0}, \mathbf{R}_{\mathbf{q q}}\left(\mathbf{H}_{\mathrm{b}}\right)\right)$ [39]. Since $\mathbf{q}$ is uncorrelated with $\mathbf{y}$ [37], the covariance matrix of $\mathbf{q}$ with $\mathbf{H}_{\mathrm{b}}$ is given as [37], [39]

$$
\mathbf{R}_{\mathbf{q q}}\left(\mathbf{H}_{\mathrm{b}}\right)=\alpha(1-\alpha) \operatorname{diag}\left(\rho \mathbf{H}_{\mathrm{b}} \mathbf{H}_{\mathrm{b}}^{H}+\mathbf{I}_{N}\right) \text {. }
$$

In the following section, we investigate a user scheduling problem based on the considered system model. 


\section{USER SCHEDULING}

In this section, we focus on zero-forcing (ZF) combining $\mathbf{W}_{\mathrm{zf}}=\mathbf{H}_{\mathrm{b}}\left(\mathbf{H}_{\mathrm{b}}^{H} \mathbf{H}_{\mathrm{b}}\right)^{-1}$ at the BS and investigate user scheduling to derive scheduling criteria and propose an algorithm by exploiting the obtained criteria. To this end, we first consider the case where the effective CSI $\mathbf{H}_{\mathrm{b}}$ is known at the BS and then extend the problem to the case where only the partial CSI is available. For lowresolution ADC systems, state-of-the-art channel estimation techniques have been developed and have shown remarkable estimation accuracy with few-bit ADCs [11], [12] or even with one-bit ADCs [8]-[10]. With the $\mathrm{ZF}$ combiner $\mathbf{W}_{\mathrm{zf}}$, the quantized signal in (6) is given as

$$
\mathbf{y}_{\mathrm{q}}^{\mathrm{zf}}=\mathbf{W}_{\mathrm{zf}}^{H} \mathbf{y}_{\mathrm{q}}=\alpha \sqrt{\rho} \mathbf{W}_{\mathrm{zf}}^{H} \mathbf{H}_{\mathrm{b}} \mathbf{s}+\alpha \mathbf{W}_{\mathrm{zf}}^{H} \mathbf{v}+\mathbf{W}_{\mathrm{zf}}^{H} \mathbf{q} .
$$

Nulling out the inter-user interference, the achievable rate of user $k$ is derived as

$$
r_{k}\left(\mathbf{H}_{\mathrm{b}}\right)=\log _{2}\left(1+\frac{\alpha^{2} \rho}{\mathbf{w}_{\mathrm{zf}, k}^{H} \mathbf{R}_{\mathbf{q q}}\left(\mathbf{H}_{\mathrm{b}}\right) \mathbf{w}_{\mathrm{zf}, k}+\alpha^{2}\left\|\mathbf{w}_{\mathrm{zf}, k}\right\|^{2}}\right)
$$

Using the achievable rate with quantization error (8), we formulate a user scheduling problem:

$$
\mathcal{P} 1: \quad \mathcal{R}\left(\mathbf{H}_{\mathrm{b}}\left(\mathcal{S}^{\star}\right)\right)=\max _{\mathcal{S} \subset\{1, \ldots, K\}:|\mathcal{S}| \leq S} \sum_{k \in \mathcal{S}} r_{k}\left(\mathbf{H}_{\mathrm{b}}(\mathcal{S})\right)
$$

where $\mathcal{S}$ represents the set of scheduled users, $\mathbf{H}_{\mathrm{b}}(\mathcal{S})$ is the beamspace channel matrix of the users in $\mathcal{S}$, and $\mathcal{R}\left(\mathbf{H}_{\mathrm{b}}(\mathcal{S})\right)$ is the sum rate of the scheduled users in $\mathcal{S}$. Unlike the user scheduling without quantization, which considers the channel orthogonality and the large channel gains, the user scheduling with the coarse quantization needs to consider an additional condition.

Remark 1. To maximize the achievable rate (8), the aggregated beamspace channel gain at each $R F$ chain $\left\|\left[\mathbf{H}_{\mathrm{b}}\right]_{i,:}\right\|^{2}$ needs to be minimized to reduce the quantization noise variance $\mathbf{R}_{\mathbf{q q}}$ in addition to forcing the channel orthogonality $\left(\mathbf{h}_{\mathrm{b}, k} \perp \mathbf{h}_{\mathrm{b}, k^{\prime}}, k \neq k^{\prime}\right)$ and maximizing the beamspace channel gain $\left\|\mathbf{h}_{\mathrm{b}, k}\right\|^{2}$, which reduces $\left\|\mathbf{w}_{\mathrm{zf}, k}\right\|^{2}$.

\section{A. Analysis of Scheduling Criteria}

We derive the scheduling criteria for channels in the beamspace based on the finding in Remark 1 to propose an efficient scheduling algorithm that solves $\mathcal{P} 1$ in $(9)$. To focus on key scheduling ingredients besides the channel magnitude, we consider the case where the magnitude of each user channel is given in the analysis, i.e., $\left\|\mathbf{h}_{\mathrm{b}, k}\right\|=\sqrt{\gamma_{k}}, \forall k$ with $\gamma_{k}>0$. Given $\left\|\mathbf{h}_{\mathrm{b}, k}\right\|=\sqrt{\gamma_{k}}, \forall k$, 
we reformulate $\mathcal{P} 1$ to the problem of finding the optimal channel matrix that maximizes the uplink sum rate to characterize the channel matrix that fully extracts scheduling gains.

$$
\mathcal{P} 2: \quad \mathcal{R}\left(\mathbf{H}_{\mathrm{b}}^{\star}\right)=\max _{\mathbf{H}_{\mathrm{b}} \in \mathbb{C}^{N \times S}} \sum_{k=1}^{S} r_{k}\left(\mathbf{H}_{\mathrm{b}}\right), \quad \text { s.t. }\left\|\mathbf{h}_{\mathrm{b}, k}\right\|=\sqrt{\gamma_{k}} \forall k .
$$

To provide geometrical interpretation for the channel matrix analysis, we further adopt the virtual channel representation [30], where each beamspace channel $\mathbf{h}_{\mathrm{b}, k}$ contains $\left(N-L_{k}\right)$ zeros and $L_{k}$ complex gains of the $L_{k}$ channel paths. We first consider the single user scheduling $(S=1)$ and derive the channel characteristics required to maximize the achievable rate for $\mathcal{P} 2$. Then, we utilize the result to derive the scheduling criteria for the multiuser scheduling case.

Lemma 1. For a single user scheduling $(S=1)$, scheduling a user who has the following channel characteristics maximizes the uplink achievable rate in $\mathcal{P} 2$ :

(i) the largest number of channel propagation paths and

(ii) equal power spread across the beamspace complex gains.

Proof. The ZF combiner for a single user becomes $\mathbf{w}_{\mathrm{zf}}=\mathbf{h}_{\mathrm{b}} /\left\|\mathbf{h}_{\mathrm{b}}\right\|^{2}$. Then, (8) is given as

$$
\begin{aligned}
\mathcal{R}\left(\mathbf{h}_{\mathrm{b}}\right) & =\log _{2}\left(1+\frac{\alpha \rho}{(1-\alpha) \frac{\mathbf{h}_{\mathrm{b}}^{H}}{\left\|\mathbf{h}_{\mathrm{b}}\right\|^{2}} \operatorname{diag}\left(\rho \mathbf{h}_{\mathrm{b}} \mathbf{h}_{\mathrm{b}}^{H}+\mathbf{I}_{N}\right) \frac{\mathbf{h}_{\mathrm{b}}}{\left\|\mathbf{h}_{\mathrm{b}}\right\|^{2}}+\frac{\alpha}{\left\|\mathbf{h}_{\mathrm{b}}\right\|^{2}}}\right) \\
& =\log _{2}\left(1+\frac{\alpha \rho\left\|\mathbf{h}_{\mathrm{b}}\right\|^{4}}{\rho(1-\alpha) \sum_{i \in \mathcal{L}}\left|h_{\mathrm{b}, i}\right|^{4}+\left\|\mathbf{h}_{\mathrm{b}}\right\|^{2}}\right)
\end{aligned}
$$

where $\mathcal{L}$ is the set of indices of non-zero complex gains in $\mathbf{h}_{\mathrm{b}}$ with $|\mathcal{L}|=L$. With the constraint of $\left\|\mathbf{h}_{\mathrm{b}}\right\|=\sqrt{\gamma}$, the problem of maximizing $\mathcal{R}\left(\mathbf{h}_{\mathrm{b}}\right)$ in 111 reduces to

$$
\min _{\mathbf{h}_{\mathrm{b}}} \sum_{i \in \mathcal{L}}\left|h_{\mathrm{b}, i}\right|^{4} \quad \text { s.t. }\left\|\mathbf{h}_{\mathrm{b}}\right\|^{2}=\gamma
$$

We use Karush-Kuhn-Tucker conditions to solve the reduced problem in (12). Let $x_{i}=\left|h_{\mathrm{b}, i}\right|^{2}$ for $i=1,2, \ldots, N$. The Lagrangian of the problem with a Lagrangian multiplier $\mu$ is given as

$$
\mathfrak{L}(\mathbf{x}, \mu)=\|\mathbf{x}\|^{2}+\mu\left(\sum_{i \in \mathcal{L}} x_{i}-\gamma\right) .
$$

By taking a derivative of $\mathfrak{L}(\mathbf{x}, \mu)$ with respect to $x_{i}$ for $i \in \mathcal{L}$ and setting it to zero, we obtain $x_{i}=-\mu / 2$. Putting it to $\sum_{i \in \mathcal{L}} x_{i}=\gamma$, we have $\mu=-2 \gamma / L$. Finally, the solution becomes

$$
x_{i}=\gamma / L, \quad i \in \mathcal{L}
$$


Under the virtual channel representation, $x_{i}$ indicates the power of the beamspace complex gains and $L$ is the number of propagation paths. Accordingly, the physical meaning of (13) is that the achievable rate for the single user case with the given channel power $\left\|\mathbf{h}_{\mathrm{b}}\right\|^{2}=\gamma$ can be maximized when the channel power $\gamma$ is evenly spread to the $L$ beamspace complex gains.

By applying the solution $\left|h_{\mathrm{b}, i}^{\star}\right|^{2}=\gamma / L$ in (13) for $i \in \mathcal{L}$, the achievable rate in (11) becomes

$$
\mathcal{R}\left(\mathbf{h}_{\mathrm{b}}^{\star}\right)=\log _{2}\left(1+\frac{\alpha \rho}{\rho(1-\alpha) / L+1 / \gamma}\right) .
$$

The quantization noise variance term in (14) decreases as $L$ increases. Therefore, the achievable rate $\mathcal{R}\left(\mathbf{h}_{\mathrm{b}}^{\star}\right)$ can be further maximized if the scheduled user channel $\mathbf{h}_{\mathrm{b}}^{\star}$ has the largest number of propagation paths with equal power distribution across the beamspace complex gains.

Unlike the conventional understanding that scheduling a user with the largest channel gain achieves the maximum achievable rate for the single user communication in the noise limited environment, Lemma 1 shows that the achievable rate is related not only to the channel magnitude $\left\|\mathbf{h}_{\mathrm{b}}\right\|$ but also to the channel structure in the beamspace when received signals are coarsely quantized. We further show that if the number of propagation paths $L$ is limited, the maximum rate for the single user case converges to a finite value as the channel magnitude increases.

Corollary 1. With the finite number of channel propagation paths $L$, the maximum achievable rate with single user scheduling converges to

$$
\mathcal{R}\left(\mathbf{h}_{\mathrm{b}}^{\star}\right) \rightarrow \log _{2}(1+\alpha L /(1-\alpha)), \quad \text { as }\left\|\mathbf{h}_{\mathrm{b}}\right\| \rightarrow \infty .
$$

Proof. The maximum achievable rate of the single user scheduling with the given $L$ and $\left\|\mathbf{h}_{\mathrm{b}}\right\|^{2}=$ $\gamma$ is derived in (14). Then, (14) converges to (15) as increasing the channel gain $(\gamma \rightarrow \infty)$.

Corollary 1 shows that the quantization error $(\alpha<1)$ limits the achievable rate to remain finite because the quantization noise variance also increases with the increase of the channel magnitude. This implies that the conventional scaling law $\log \log K$ [20] cannot be met in the low-resolution ADCs regime. Accordingly, as the SNR increases, mitigation of the quantization error becomes a more critical problem that needs to be considered in user scheduling.

Now, we investigate the multiuser scheduling for the case where $\sum_{k=1}^{S} L_{\mathcal{S}(k)} \leq N$. Here, $\mathcal{S}(k)$ is the $k$ th scheduled user. This condition is relevant to mmWave channels where the number of channel paths $L_{k}$ is presumably very small [42]. We solve the problem $\mathcal{P} 2$ to characterize the channel properties that maximize the scheduling gain. Theorem 1 shows the structural scheduling criteria of channels to maximize the sum rate in $\mathcal{P} 2$ for the considered case. 
Theorem 1. For the case where $\sum_{k=1}^{S} L_{\mathcal{S}(k)} \leq N$, scheduling a set of users $\mathcal{S}$ that satisfies the following channel characteristics maximizes the uplink sum rate in $\mathcal{P} 2$.

(i) Unique AoAs at the receiver for the channel propagation paths of each scheduled user:

$$
\mathcal{L}_{\mathcal{S}(k)} \cap \mathcal{L}_{\mathcal{S}\left(k^{\prime}\right)}=\emptyset \text { if } k \neq k^{\prime}
$$

where $\mathcal{L}_{\mathcal{S}(k)}$ represents the set of indices of non-zero complex gains in $\mathbf{h}_{\mathrm{b}, \mathcal{S}(k)}$.

(ii) Equal power spread across the beamspace complex gains within each user channel:

$$
\left|h_{\mathrm{b}, i, \mathcal{S}(k)}\right|=\sqrt{\gamma_{\mathcal{S}(k)} / L_{\mathcal{S}(k)}} \text { for } i \in \mathcal{L}_{\mathcal{S}(k)} .
$$

Proof. We take a two-stage maximization approach and show the sufficient conditions for maximizing the sum rate in $\mathcal{P} 2$ with the constraint of $\sum_{k=1}^{S} L_{\mathcal{S}(k)} \leq N$. Using the diagonal structure of $\mathbf{R}_{\mathbf{q q}}$ as shown in (7), we rewrite (8) in a simpler form as

$$
r_{k}\left(\mathbf{H}_{\mathrm{b}}\right)=\log _{2}\left(1+\frac{\alpha \rho}{\rho(1-\alpha) \mathbf{w}_{\mathrm{zf}, k}^{H} \operatorname{diag}\left(\mathbf{H}_{\mathrm{b}} \mathbf{H}_{\mathrm{b}}^{H}\right) \mathbf{w}_{\mathrm{zf}, k}+\left\|\mathbf{w}_{\mathrm{zf}, k}\right\|^{2}}\right) .
$$

In the first stage, we focus on minimizing $\left\|\mathbf{w}_{\mathrm{zf}, k}\right\|^{2}$ in (18). When user channels are orthogonal, $\mathbf{h}_{\mathrm{b}, k} \perp \mathbf{h}_{\mathrm{b}, k^{\prime}}$ for $k \neq k^{\prime}$, we have $\mathbf{w}_{\mathrm{zf}, k}=\mathbf{h}_{\mathrm{b}, k} /\left\|\mathbf{h}_{\mathrm{b}, k}\right\|^{2}$. Since $\mathbf{w}_{\mathrm{zf}, k}$ with minimum norm is known as $\mathbf{w}_{\mathrm{zf}, k}=\mathbf{h}_{\mathrm{b}, k} /\left\|\mathbf{h}_{\mathrm{b}, k}\right\|^{2}$, $\left\|\mathbf{w}_{\mathrm{zf}, k}\right\|^{2}$ can be minimized with the orthogonality condition.

In the second stage, we minimize the achievable rate of (18) by imposing the orthogonality condition from the first stage as follows:

$$
\begin{aligned}
r_{k}\left(\mathbf{H}_{\mathrm{b}} \mid \mathbf{h}_{\mathrm{b}, k} \perp \mathbf{h}_{\mathrm{b}, k^{\prime}}\right) & \stackrel{(a)}{=} \log _{2}\left(1+\frac{\alpha \rho\left\|\mathbf{h}_{\mathrm{b}, k}\right\|^{4}}{\rho(1-\alpha) \mathbf{h}_{\mathrm{b}, k}^{H} \operatorname{diag}\left(\mathbf{H}_{\mathrm{b}} \mathbf{H}_{\mathrm{b}}^{H}\right) \mathbf{h}_{\mathrm{b}, k}+\left\|\mathbf{h}_{\mathrm{b}, k}\right\|^{2}}\right) \\
& =\log _{2}\left(1+\frac{\alpha \rho \gamma_{k}^{2}}{\rho(1-\alpha) \sum_{i \in \mathcal{L}_{k}}\left|h_{\mathrm{b}, i, k}\right|^{2}\left(\left|h_{\mathrm{b}, i, k}\right|^{2}+\sum_{u \neq k}\left|h_{\mathrm{b}, i, u}\right|^{2}\right)+\gamma_{k}}\right) \\
& \stackrel{(b)}{\leq} \log _{2}\left(1+\frac{\alpha \rho \gamma_{k}^{2}}{\rho(1-\alpha) \sum_{i \in \mathcal{L}_{k}}\left|h_{\mathrm{b}, i, k}\right|^{4}+\gamma_{k}}\right) \\
& \stackrel{(c)}{\leq} \log _{2}\left(1+\frac{\alpha \rho}{\rho(1-\alpha) / L_{k}+1 / \gamma_{k}}\right) .
\end{aligned}
$$

The equality (a) is from $\mathbf{w}_{\mathrm{zf}, k}=\mathbf{h}_{\mathrm{b}, k} /\left\|\mathbf{h}_{\mathrm{b}, k}\right\|^{2}$. The equality in (b) holds if and only if $\left|h_{\mathrm{b}, i, u}\right|=0$, $\forall u \neq k$ and $i \in \mathcal{L}_{k}$. This implies that each user needs to have channel paths with unique AoAs to maximize the achievable rate. Note that (20) is equivalent to the achievable rate of the single user 
scheduling in (11) due to the channel orthogonality and unique AoA conditions. Consequently, applying Lemma 1, we have the inequality (c) which comes from the fact that 20) is maximized when $\left|h_{\mathrm{b}, i, k}\right|=\sqrt{\gamma_{k} / L_{k}}$ for $i \in \mathcal{L}_{k}$, i.e., channel power is spread evenly across the beamspace complex gains within each user channel. The upper bound in (21) is equivalent to the maximum achievable rate for the single user case in (14). Therefore, (21) is also the maximum achievable rate of each user for the problem $\mathcal{P} 2$, which also maximizes the sum rate in $\mathcal{P} 2$.

Throughout the proof, we have shown that the derived conditions: the orthogonality, the unique AoA, and the equal power spread conditions are sufficient to maximize the sum rate in $\mathcal{P} 2$ for the case of $\sum_{k=1}^{S} L_{\mathcal{S}(k)} \leq N$. Since, the unique AoA condition implies the orthogonality, only the unique AoA and equal power spread conditions are required to be satisfied by the beamspace channel matrix $\mathbf{H}_{\mathrm{b}}$ for maximizing the uplink sum rate. This completes the proof.

Distinguished from conventional channels, there are channel orthogonality cases related to mmWave massive MIMO communications: (a) asymptotic orthogonality of array steering vectors across different angles [52], (b) orthogonality of beamspace channel sub-vectors having common AoAs, and (c) orthogonality of array steering vectors in (2) with angle offsets of multiples of $2 / M$ [28]. Note that the first condition in (16) particularly emphasizes the third case (c) which forces the beamspace channel orthogonality and further minimizes the aggregated channel gain at each RF chain by avoiding overlap between channel gains in the same AoA, which reduces the quantization noise variance as discussed in Remark 1. The second condition in (17) also minimizes the aggregated channel gain by evenly spreading the channel power across the beamspace gains, and thus, reduces the quantization error. Consequently, Theorem 1 emphasizes the importance of the channel structure in maximizing the sum rate under coarse quantization, while conventional user scheduling approaches ignore such criteria. Therefore, we propose a quantization-aware scheduling algorithm based on the criteria in Theorem 1. Although the scheduling criteria in Theorem 1 is derived under the condition of $\sum_{k=1}^{S} L_{\mathcal{S}(k)} \leq N$, we show that the proposed algorithm which exploits the criteria still achieves higher performance compared to conventional algorithms for $\sum_{k=1}^{S} L_{\mathcal{S}(k)}>N$ in Section $\mathrm{V}$

\section{B. Proposed Algorithm}

In this subsection, we propose a user scheduling algorithm with low complexity by using the criteria in Theorem 1. Adopting a greedy manner, the proposed algorithms make it possible 


\section{Algorithm 1: Channel Structure-based Scheduling (CSS)}

1 Initialization: $\mathcal{K}_{1}=\{1, \ldots, K\}, \mathcal{S}=\phi$, and $i=1$.

2 for $k=1: K$ do

$3 \quad$ BS stores $N_{b} \geq L_{k}$ indices of dominant spatial angles of $\mathbf{h}_{\mathrm{b}, k}$ in $\mathcal{B}_{k}$.

4 Iteration: while $i \leq S$ and $\mathcal{K}_{i} \neq \emptyset$ do

5 for $k \in \mathcal{K}_{i}$ do

6

BS computes approximated SINR of user $k, \operatorname{SINR}_{k}\left(\mathbf{H}_{\mathrm{b}}(\mathcal{S} \cup\{k\})\right)$ in 25.

BS schedules user who has the largest SINR as

$$
\mathcal{S}(i)=\underset{k \in \mathcal{K}_{i}}{\operatorname{argmax}} \operatorname{SINR}_{k}\left(\mathbf{H}_{\mathrm{b}}(\mathcal{S} \cup\{k\})\right)
$$

and updates scheduled user set $\mathcal{S}=\mathcal{S} \cup\{\mathcal{S}(i)\}$.

10 return Scheduled user set $\mathcal{S}$;

to schedule users without examining all combinations of users. At each iteration, the proposed algorithm schedules a user and reduces the size of a user candidate set $\mathcal{K}$ through filtering. To extract user diversity, the algorithm filter the user set $\mathcal{K}$ by enforcing semi-orthogonality between scheduled user channels, not perfect orthogonality. In addition to the scheduling criteria in Theorem 11, we also apply the orthogonality condition in (19) for the filtering to provide higher precision in the semi-orthogonality.

Algorithm 1 describes the proposed scheduling method, called channel structure-based scheduling (CSS). After each user selection, the proposed algorithm filters the user candidate set $\mathcal{K}$ by leveraging the orthogonality condition in (19) as in [19] by utilizing (23)

$$
\mathbf{f}_{\mathcal{S}(i)}=\mathbf{h}_{\mathrm{b}, \mathcal{S}(i)}-\sum_{j=1}^{i-1} \frac{\mathbf{f}_{\mathcal{S}(j)}^{H} \mathbf{h}_{\mathrm{b}, \mathcal{S}(i)}}{\left\|\mathbf{f}_{\mathcal{S}(j)}\right\|^{2}} \mathbf{f}_{\mathcal{S}(j)}=\left(\mathbf{I}-\sum_{j=1}^{i-1} \frac{\mathbf{f}_{\mathcal{S}(j)} \mathbf{f}_{\mathcal{S}(j)}^{H}}{\left\|\mathbf{f}_{\mathcal{S}(j)}\right\|^{2}}\right) \mathbf{h}_{\mathrm{b}, \mathcal{S}(i)}
$$

where $\mathbf{f}_{\mathcal{S}(i)}$ is the component of $\mathbf{h}_{\mathrm{b}, \mathcal{S}(i)}$ that is orthogonal to subspace $\operatorname{span}\left\{\mathbf{f}_{\mathcal{S}(1)}, \ldots, \mathbf{f}_{\mathcal{S}(i-1)}\right\}$. Unlike the algorithm in [19] which computes the orthogonal component $\mathbf{f}_{k}$ for the entire users in the candidate set, the proposed CSS algorithm calculates $\mathbf{f}_{\mathcal{S}(i)}$ only for the currently scheduled user $\mathcal{S}(i)$. The algorithm also enforces additional spatial orthogonality in the beamspace to the filtered set as in [28] by modifying the unique AoA condition in (16). Since there can exist phase 
offsets that lead to more than $L_{k}$ dominant channel gains in $\mathbf{h}_{\mathrm{b}, k}$ due to the quantized angles of the analog combiner, the algorithm stores $N_{b} \geq L_{k}$ indices of dominant spatial angles in $\mathcal{B}_{k}$ and filters the user set $\mathcal{K}$ by removing users whose angle indices in $\mathcal{B}_{k}$ show more than $N_{\mathrm{OL}}$ overlaps with those of the scheduled user in $\mathcal{B}_{\mathcal{S}(i)}$. The semi-orthogonality filtering becomes

$$
\mathcal{K}_{i+1}=\left\{k \in \mathcal{K}_{i} \backslash\{\mathcal{S}(i)\}\left|\frac{\left|\mathbf{f}_{\mathcal{S}(i)}^{H} \mathbf{h}_{\mathrm{b}, k}\right|}{\left\|\mathbf{f}_{\mathcal{S}(i)}\right\|\left\|\mathbf{h}_{\mathrm{b}, k}\right\|}<\epsilon,\right| \mathcal{B}_{\mathcal{S}(i)} \cap \mathcal{B}_{k} \mid \leq N_{\mathrm{OL}}\right\} .
$$

These filtering operations not only reduce the size of the user set $\mathcal{K}$, but also offer semiorthogonality between the scheduled users in $\mathcal{S}$ and the candidate users in $\mathcal{K}$. As a result, the filtering leads the ZF combiner to be approximated as $\mathbf{w}_{\mathrm{zf}, k} \approx \mathbf{h}_{\mathrm{b}, k} /\left\|\mathbf{h}_{\mathrm{b}, k}\right\|^{2}$ for a user $k \in \mathcal{K}$, and we can approximate the SINR of user $k \in \mathcal{K}$ with previously scheduled users in $\mathcal{S}$ as

$$
\operatorname{SINR}_{k}\left(\mathbf{H}_{\mathrm{b}}(\mathcal{S} \cup\{k\})\right) \approx \frac{\alpha \rho\left\|\mathbf{h}_{\mathrm{b}, k}\right\|^{4}}{(1-\alpha) \mathbf{h}_{\mathrm{b}, k}^{H} \mathbf{D}\left(\mathbf{H}_{\mathrm{b}}(\mathcal{S} \cup\{k\})\right) \mathbf{h}_{\mathrm{b}, k}}
$$

where $\mathbf{D}\left(\mathbf{H}_{\mathrm{b}}(\mathcal{S} \cup\{k\})\right)=\operatorname{diag}\left(\rho \mathbf{H}_{\mathrm{b}}(\mathcal{S} \cup\{k\}) \mathbf{H}_{\mathrm{b}}(\mathcal{S} \cup\{k\})^{H}+\frac{1}{1-\alpha} \mathbf{I}_{N}\right)$. For a scheduling measure, the proposed algorithm adopts the approximated SINR (25) to incorporate the scheduling criteria in Theorem 1 with the channel magnitude and orthogonality ${ }^{2}$. At each iteration, the algorithm schedules the user who has the largest SINR among the users in $\mathcal{K}$ as shown in 22 . Using the approximated SINR (25) for the selection measure greatly reduces the computational complexity by avoiding the matrix inversion for computing the $\mathrm{ZF}$ combiner $\mathbf{W}_{\mathrm{zf}}$.

To provide a reference in sum rate performance, we also propose a high-complexity and highperformance greedy algorithm which schedules the user who achieves the highest sum rate at each iteration as shown in Algorithm 2. At each iteration, the greedy algorithm computes sum rate in (8), i.e., the algorithm computes the exact SINR for scheduled users in $\mathcal{S}_{\mathrm{G}}$ and a candidate user $k, \forall k \in \mathcal{K}_{\mathrm{G}, i}$. Thus, the algorithm carries the huge burden of computing a matrix inversion $\left|\mathcal{K}_{\mathrm{G}, i}\right|$ times at each selection. At the $i$ th stage, the greedy algorithm computes the achievable rate in (8) $\left|\mathcal{K}_{\mathrm{G}, i}\right| \times i$ times and compares the derived $\left|\mathcal{K}_{\mathrm{G}, i}\right|$ sum rates, whereas the CSS algorithm only computes the approximated SINR in $25\left|\mathcal{K}_{i}\right|$ times and compares $\left|\mathcal{K}_{i}\right|$ SINRs. Moreover, unlike the greedy algorithm, the CSS algorithm reduces the size of the user set $\mathcal{K}_{i}$ by filtering in (24) at each iteration. This leads to $\left|\mathcal{K}_{i}\right| \ll\left|\mathcal{K}_{\mathrm{G}, i}\right|$, and the gap $\left|\mathcal{K}_{\mathrm{G}, i}\right|-\left|\mathcal{K}_{i}\right|$ will increase with iteration; the CSS algorithm becomes more efficient with larger $K$ and /or $S$.

\footnotetext{
${ }^{2}$ By treating the approximate SINR as the true SINR and following the technique used in [19| and [28], the proposed method can be incorporated with the proportional fairness (PF) policy [43] for fairness-aware scheduling in a heterogeneous system.
} 


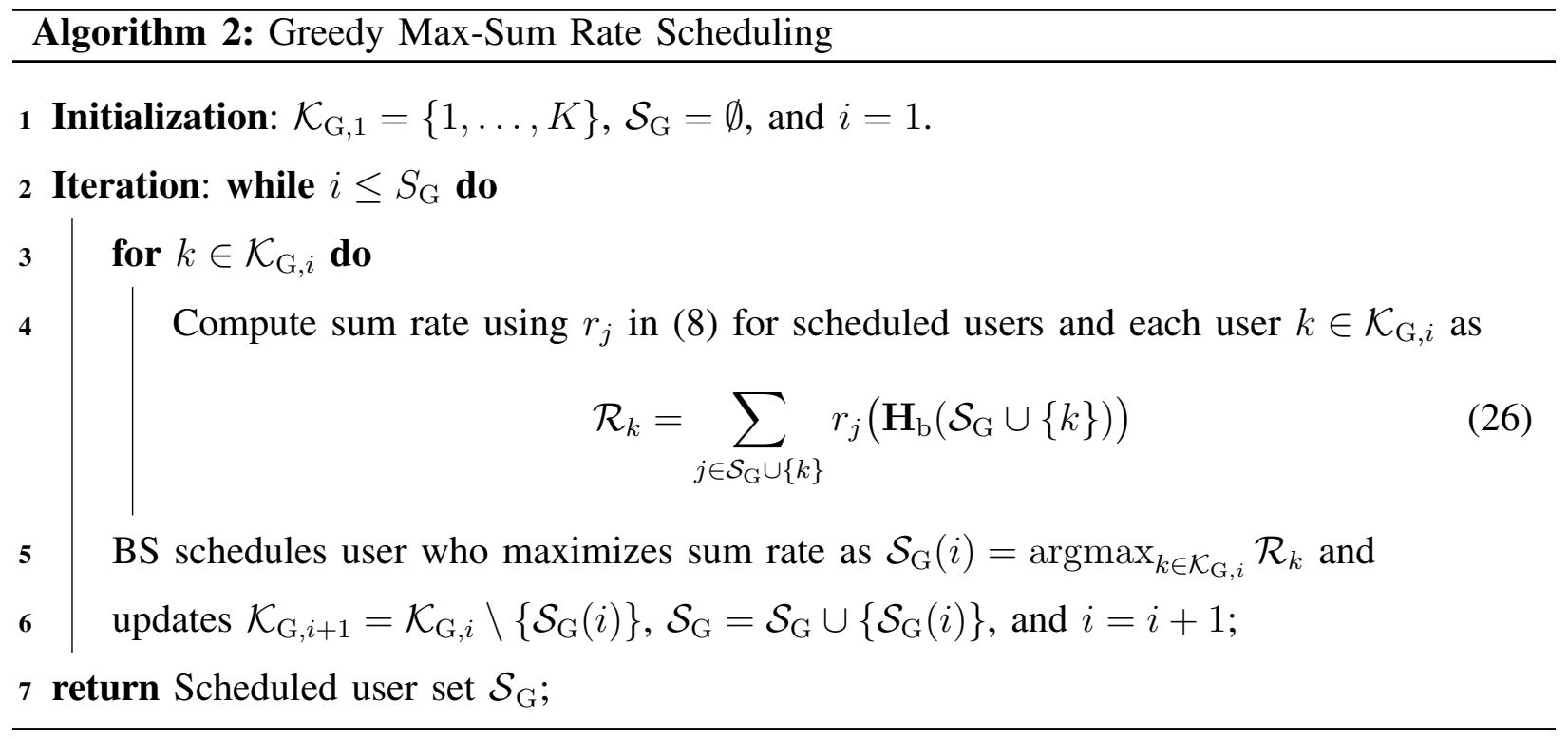

Remark 2. The proposed algorithm can be applied to an orthogonal frequency division multiplexing (OFDM) system for a wideband channel case. Since we consider the system with a given analog combiner, the proposed algorithm can be performed independently for each subcarrier index $i$. However, the structure of the quantization noise $\mathbf{q}[i]$ in the wideband OFDM system becomes different from that of the narrowband system so that the spatial filtering in the proposed user scheduling algorithm may not be desirable. Nonetheless, the approximated SINR can still be applicable with the semi-orthogonality filtering by computing the quantization noise variance for each subcarrier $i$ of the OFDM system $\mathbf{R}_{\mathbf{q q}}[i]$. Thus, the BS can perform the proposed algorithms to schedule users to be served on each subcarrier by relaxing the spatial filtering.

We note that the proposed method schedules users with minimum overlap among quantized AoAs of user channels to satisfy the derived scheduling criterion (i) in Theorem 1. Accordingly, by using the proposed scheduling method, the beamforming-based Doppler effect reduction techniques such as a per-beam synchronization approach in [?] can be performed at the BS since the BS can see each beam with a single dedicated user signal with large channel gains and possibly with other user signals with negligible channel gains. Therefore, the proposed scheduling method can provide potential benefit in reducing Doppler effect when jointly used with Doppler effect mitigation techniques at the BS. 


\section{Beam Training-Based Channel Acquisition}

Assuming time-division duplex communications, we briefly provide an example of extension our algorithm to a practical system where the BS uses beam training and receives channel quality indicators (CQIs) from users. A procedure of beam training and CQI feedback can be as follows:

1) The BS constructs a set of $N_{s} \geq N$ beam vectors $\left\{\mathbf{a}\left(\bar{\vartheta}_{1}\right), \ldots, \mathbf{a}\left(\bar{\vartheta}_{N_{s}}\right)\right\}$ with the angles within the angles of the analog combiner $\tilde{\mathbf{A}}$, i.e., there exists $i$ such that $\bar{\vartheta}_{n} \in\left[\vartheta_{i}-1 / M, \vartheta_{i}+1 / M\right]$, $\forall n$, where $\vartheta_{i}$ is the spatial angle of the $i$ th analog beamformer. Then, the BS transmits each beam of the set in time to all users in the cell during a training phase.

2) Each user $k$ can estimate the channel gain corresponding to each beam and have the estimate of $\mathbf{h}_{k}^{H} \tilde{\mathbf{A}}=\mathbf{h}_{\mathrm{b}, k}^{H}$ at the end of the beam training. From the sparsity of the mmWave channel, few elements of $\mathbf{h}_{\mathrm{b}, k}$ have non-negligible beam gains and we can implement an efficient feedback method that exploits the sparsity of the effective channel $\mathbf{h}_{\mathrm{b}, k}$ as described in [44]. For instance, each user can feed back the beam indices of the non-negligible beam gains and their corresponding channel coefficients in a quantized form to the BS.

3) After the feedback from all users is over, the BS can create an estimate of $\mathbf{H}_{b}$ with the feedback information by simply padding zeros in the unreported beam indices. Then, the BS can directly apply the proposed scheduling algorithm by using the estimated channel.

The estimation error with the CQI feedback is expected to degrade both the proposed algorithm and conventional scheduling algorithms since full CSI is required for all approaches. We leave the analysis of the imperfect CSI case for a future work as it is beyond the scope of our work.

\section{User Scheduling With Partial Channel Information}

In this section, we propose a user scheduling algorithm when only partial CSI is known at the BS since it can be challenging to obtain reliable instantaneous CSI estimates for entire users as the number of antennas or users becomes large. A reasonable alternative is to use slowly-varying channel characteristics, in particular, AoAs of mmWave channels [31]; AoAs persist over longer than the coherence time of mmWave channels, and mmWave channels have a limited number of AoAs. In this regard, by using the AoA knowledge, the proposed algorithm can greatly reduce the burden of estimating instantaneous full CSI at each channel coherence time. After scheduling, we assume that the BS acquires the effective CSI of the scheduled users for decoding. 


\section{A. Proposed Algorithm}

According to (1), the channel $\mathbf{h}_{k}$ lies in the subspace spanned by its array response vectors, i.e., $\mathbf{h}_{k} \in \operatorname{span}\left\{\mathbf{a}\left(\phi_{k, 1}\right), \ldots, \mathbf{a}\left(\phi_{k, L_{k}}\right)\right\}$. To measure the separation between the subspaces, we adopt chordal distance which measures the angle between the subspaces. In the initialization phase, the algorithm removes users whose AoAs are not in the range of angles of RF chains (reduced range of angles) $]^{3}$ from the initial candidate user set $\mathcal{K}_{\mathrm{cd}, 1}$. In the scheduling phase, a first user is scheduled by randomly selecting a user among the set of users with the most AoAs in the reduced range of angles. To schedule a next user, the algorithm updates the candidate user set $\mathcal{K}_{\mathrm{cd}, i}$ by filtering users whose chordal distance is shorter than the threshold $d_{\text {th }}$ to impose semi-orthogonality among scheduled users. Due to the filtering, the remaining users in $\mathcal{K}_{\mathrm{cd}, i+1}$ are guaranteed to have a certain level of orthogonality with the scheduled users $\mathcal{S}(j)$ for $j=1,2, \ldots, i-1$. Then, the algorithm schedules the user with the longest chordal distance among the remaining users with the most AoAs in the reduced range of angles.

To this end, we generate the matrix of array response vectors for each user by exploiting the AoA knowledge as $\mathbf{A}_{k}=\left[\mathbf{a}\left(\phi_{k, \mathcal{V}_{k}(1)}\right), \ldots, \mathbf{a}\left(\phi_{k, \mathcal{V}_{k}\left(V_{k}\right)}\right)\right]$ where $\mathcal{V}_{k}$ is the set of AoAs indices within the reduced range of angles for user $k$ and $V_{k}=\left|\mathcal{V}_{k}\right|$. Let $\mathcal{A}_{k}=\operatorname{span}\left\{\mathbf{A}_{k}\right\}$ is the subspace for user $k$. The chordal distance between the two subspaces $\left(\mathcal{A}_{k}, \mathcal{A}_{k^{\prime}}\right)$ is defined as $d_{\mathrm{cd}}\left(k, k^{\prime}\right)=\sqrt{\sum_{\ell=1}^{L_{\min }} \sin ^{2} \theta_{\ell}}$ where $L_{\text {min }}=\min \left\{L_{k}, L_{k^{\prime}}\right\}$ and $\theta_{\ell} \leq \pi / 2$ is the principal angle between $\mathcal{A}_{k}$ and $\mathcal{A}_{k^{\prime}}$. Let $\mathrm{Q}_{k}$ be the unitary matrix whose columns are orthonormal basis vectors of $\mathcal{A}_{k}$. According to [45], we rewrite $d_{\mathrm{cd}}\left(k, k^{\prime}\right)$ as $d_{\mathrm{cd}}\left(k, k^{\prime}\right)=\sqrt{L_{\min }-\operatorname{tr}\left(\mathbf{Q}_{k}^{H} \mathbf{Q}_{k^{\prime}} \mathbf{Q}_{k^{\prime}}^{H} \mathbf{Q}_{k}\right)}$. The proposed chordal distance-based user scheduling method is described in Algorithm 3 .

Let $\tilde{\mathbf{h}}_{k}=\sqrt{\frac{M}{L_{k}}} \sum_{i \in \mathcal{V}_{k}} g_{k, i} \mathbf{a}\left(\phi_{k, i}\right)$. Then, the algorithm provides an opportunity to schedule users with nearly $\tilde{\mathbf{h}}_{k} \perp \tilde{\mathbf{h}}_{k^{\prime}}$ while the effective channel that the BS sees is the beamspace channel $\mathbf{h}_{\mathrm{b}, k}=\mathbf{W}_{\mathrm{RF}}^{H} \mathbf{h}_{k}$. Since the AoAs $\phi_{k, i}, i \in \mathcal{V}_{k}$ are in the range of angles of RF chains, $\tilde{\mathbf{h}}_{k}$ can be regarded to be in the subspace of $\mathbf{W}_{\mathrm{RF}}$, i.e., almost $\tilde{\mathbf{h}}_{k} \in \operatorname{span}\left\{\mathbf{W}_{\mathrm{RF}}\right\}$ Accordingly, using $\mathbf{W}_{\mathrm{RF}}^{H} \mathbf{W}_{\mathrm{RF}}=\mathbf{I}_{N}$ which comes from the definition i.e., a sub-matrix of the DFT matrix $\mathbf{W}_{\mathrm{RF}}=\tilde{\mathbf{A}}$, we can rewrite $\tilde{\mathbf{h}}_{k}$ as

$$
\tilde{\mathbf{h}}_{k} \approx \mathbf{W}_{\mathrm{RF}}\left(\mathbf{W}_{\mathrm{RF}}^{H} \mathbf{W}_{\mathrm{RF}}\right)^{-1} \mathbf{W}_{\mathrm{RF}}^{H} \tilde{\mathbf{h}}_{k}=\mathbf{W}_{\mathrm{RF}} \mathbf{W}_{\mathrm{RF}}^{H} \tilde{\mathbf{h}}_{k}
$$

\footnotetext{
${ }^{3}$ The range of angles of RF chains indicates the set of angles corresponding to $\bigcup_{i}\left\{\vartheta:\left|\vartheta-\vartheta_{i}\right|<\frac{1}{M}\right\}$, i.e., the AoAs in the reduced range of angles are $\varphi_{k, \ell} \in \bigcup_{i}\left\{\vartheta:\left|\vartheta-\vartheta_{i}\right|<\frac{1}{M}\right\}$.

${ }^{4}$ If the AoAs of $\tilde{\mathbf{h}}_{k}$ exactly align with the quantized angles of the analog combiner, $\tilde{\mathbf{h}}_{k}$ perfectly lies in the subspace of $\mathbf{W}_{\mathrm{RF}}$.
} 


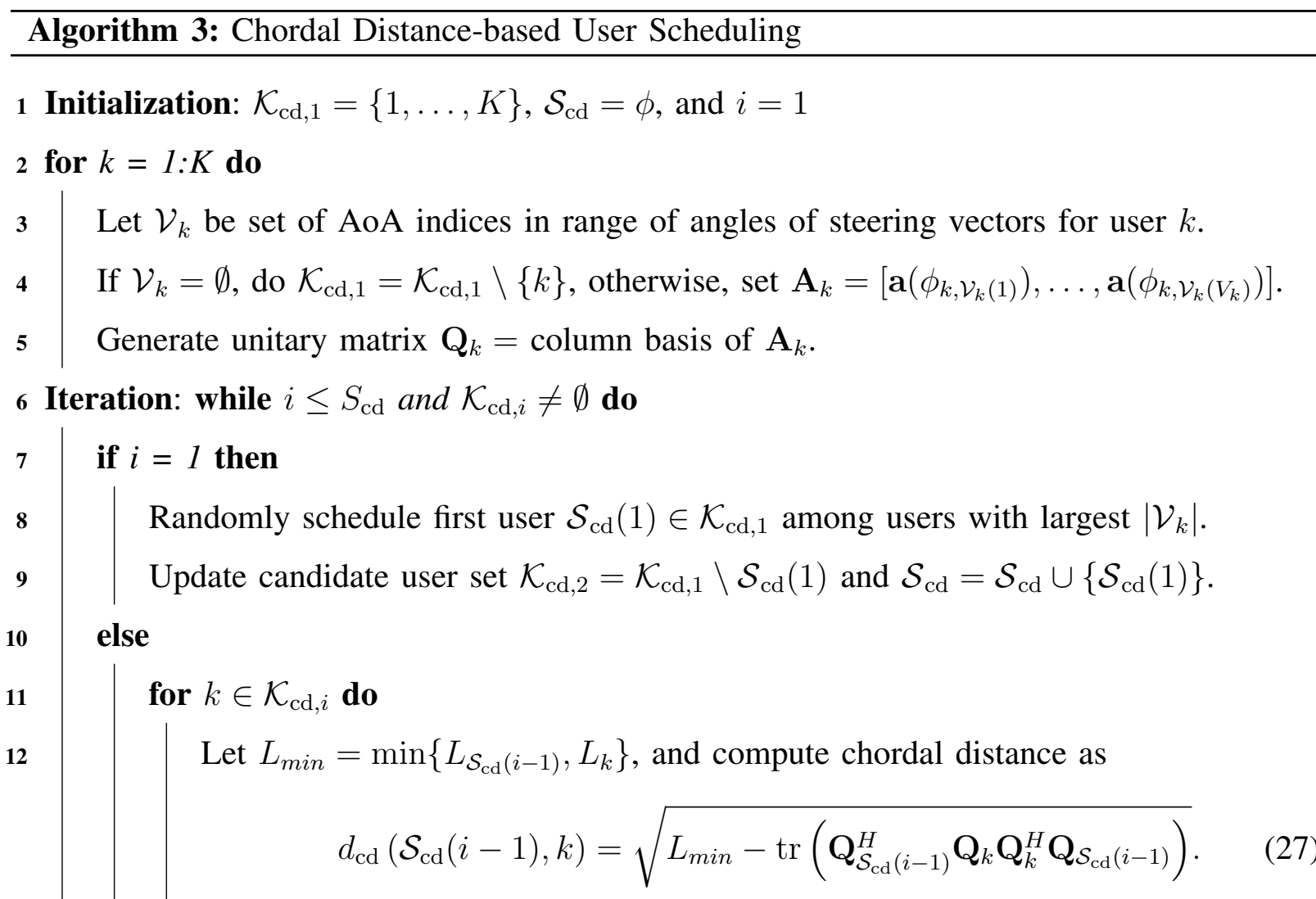

13

14

15

16

17 return Scheduled user set $\mathcal{S}_{\text {cd }}$;

Set $i=i+1$;

Filter candidate user set based on chordal distance computed in (27)

$$
\mathcal{K}_{\mathrm{cd}, i+1}=\left\{k \in \mathcal{K}_{\mathrm{cd}, i} \mid d_{\mathrm{cd}}\left(\mathcal{S}_{\mathrm{cd}}(i-1), k\right) / \sqrt{L_{\min }}>d_{\mathrm{th}}\right\}
$$

Let $\mathcal{U}$ be set of users with largest $\left|\mathcal{V}_{k}\right|, \forall k \in \mathcal{K}_{\mathrm{cd}, i+1}$. Schedule user in $\mathcal{U}$ as

$$
\mathcal{S}_{\mathrm{cd}}(i)=\underset{k \in \mathcal{U}}{\operatorname{argmax}} d_{\mathrm{cd}}\left(\mathcal{S}_{\mathrm{cd}}(i-1), k\right) .
$$

Then, update $\mathcal{K}_{\mathrm{cd}, i+1}=\mathcal{K}_{\mathrm{cd}, i+1} \backslash\left\{\mathcal{S}_{\mathrm{cd}}(i)\right\}$ and $\mathcal{S}_{\mathrm{cd}}=\mathcal{S}_{\mathrm{cd}} \cup\left\{\mathcal{S}_{\mathrm{cd}}(i)\right\}$.

In addition, we have $\mathbf{h}_{\mathrm{b}, k}=\mathbf{W}_{\mathrm{RF}}^{H} \mathbf{h}_{k} \approx \mathbf{W}_{\mathrm{RF}}^{H} \tilde{\mathbf{h}}_{k}$ as the impact of $\mathbf{a}\left(\phi_{k, j}\right), \forall j \notin \mathcal{V}_{k}$ on the beam domain channel $\mathbf{h}_{b, k}$ is relatively small compared to that of $\mathbf{a}\left(\phi_{k, i}\right), \forall i \in \mathcal{V}_{k}$ after analog combining. In this regard, as the algorithm gives $\tilde{\mathbf{h}}_{k} \perp \tilde{\mathbf{h}}_{k^{\prime}}$, we can nearly have $\mathbf{h}_{\mathrm{b}, k} \perp \mathbf{h}_{\mathrm{b}, k^{\prime}}$ by

$$
\epsilon=\tilde{\mathbf{h}}_{k}^{H} \tilde{\mathbf{h}}_{k^{\prime}} \stackrel{(a)}{\approx} \tilde{\mathbf{h}}_{k}^{H} \mathbf{W}_{\mathrm{RF}} \mathbf{W}_{\mathrm{RF}}^{H} \mathbf{W}_{\mathrm{RF}} \mathbf{W}_{\mathrm{RF}}^{H} \tilde{\mathbf{h}}_{k^{\prime}}=\tilde{\mathbf{h}}_{k}^{H} \mathbf{W}_{\mathrm{RF}} \mathbf{W}_{\mathrm{RF}}^{H} \tilde{\mathbf{h}}_{k^{\prime}} \stackrel{(b)}{\approx} \mathbf{h}_{\mathrm{b}, k}^{H} \mathbf{h}_{\mathrm{b}, k^{\prime}}
$$

where $(a)$ is from 30 and $(b)$ is from $\mathbf{h}_{\mathrm{b}, k} \approx \mathbf{W}_{\mathrm{RF}}^{H} \tilde{\mathbf{h}}_{k}$. Thus, the proposed algorithm guarantees 
a certain level of orthogonality between the beamspace channels of the scheduled users.

As discussed in Section III-C, the beam indices for non-negligible channel gains can be obtained by using CQI feedback, i.e., AoAs can be estimated for each user. When the capacity of amount of feedback is limited and small, such beam index-only feedback which requires only few integer numbers can be applied to faciliate the proposed chordal distance-based algorithm.

\section{B. Ergodic Rate Analysis}

We now analyze the performance of the chordal distance-based algorithm in ergodic rate. We focus on the case where each channel has a single propagation path, which corresponds to the sparse nature of mmWave channels [27], and the number of RF chains are equal to the number of antennas $N=M$ in the analysis.

Remark 3. When there is a single path for each user channel, the filtering in 28) reduces to $\mathcal{K}_{\mathrm{cd}, i+1}=\left\{k \in \mathcal{K}_{\mathrm{cd}, i}|| \mathbf{a}^{H}\left(\phi_{\mathcal{S}(i-1)}\right) \mathbf{a}\left(\phi_{k}\right) \mid<\epsilon_{\mathrm{th}}\right\}$ where $\epsilon_{\mathrm{th}} \ll 1$, and the scheduling problem in (29) becomes $\mathcal{S}_{\mathrm{cd}}(i)=\operatorname{argmin}_{k \in \mathcal{K}_{\mathrm{cd}, i+1}}\left|\mathbf{a}^{H}\left(\phi_{\mathcal{S}(i-1)}\right) \mathbf{a}\left(\phi_{k}\right)\right|$.

Based on Remark 3, we derive closed-form expressions of the ergodic sum rate for two different cases: (1) AoAs of channels exactly align with the quantized angles of the analog combiner, and (2) channels have arbitrary AoAs regardless of the quantized angles of the analog combiner. For the first case, there is no channel leakage in the beamspace and thus, it is often considered as a more favorable channel condition since it improves communication performance such as channel estimation accuracy [18] and achievable rate [36], [47].

Proposition 1. When AoAs of channels exactly align with the quantized angles of the analog combiner with a single propagation path, the ergodic sum rate for $\left|\mathcal{S}_{\mathrm{cd}}\right|=S$ scheduled users with the proposed chordal distance-based scheduling algorithm is derived as

$$
\overline{\mathcal{R}}_{1}=\frac{S}{\ln 2}\left(e^{\frac{1}{\rho M}} \Gamma\left(0, \frac{1}{\rho M}\right)-e^{\frac{1}{\rho(1-\alpha) M}} \Gamma\left(0, \frac{1}{\rho(1-\alpha) M}\right)\right)
$$

where $\Gamma(a, z)$ is an incomplete gamma function defined as $\Gamma(a, z)=\int_{z}^{\infty} t^{a-1} e^{-t} d t$.

Proof. See Appendix A.

Corollary 2. The derived ergodic rate (31) can be expressed as the sum of the ergodic rate without quantization error $\overline{\mathcal{R}}_{\text {inf }}$ and the ergodic rate loss due to quantization error $\overline{\mathcal{R}}_{\text {loss }}(\alpha)$

$$
\overline{\mathcal{R}}_{1}=\overline{\mathcal{R}}_{\text {inf }}+\overline{\mathcal{R}}_{\text {loss }}(\alpha)
$$


where $\overline{\mathcal{R}}_{\text {inf }}=\frac{S}{\ln 2} e^{\frac{1}{\rho M}} \Gamma\left(0, \frac{1}{\rho M}\right)$ and $\overline{\mathcal{R}}_{\text {loss }}(\alpha)=-\frac{S}{\ln 2} e^{\frac{1}{\rho(1-\alpha) M}} \Gamma\left(0, \frac{1}{\rho(1-\alpha) M}\right)$.

Proof. We can remove the quantization error term in (36) by having $\alpha=1$. Then, we have

$$
\mathbb{E}\left[\log _{2}\left(1+\rho\left\|\mathbf{h}_{\mathrm{b}, k}\right\|^{2}\right)\right]=\frac{1}{\ln 2} e^{\frac{1}{\rho M}} \Gamma\left(0, \frac{1}{\rho M}\right)
$$

as $\frac{1}{M}\left\|\mathbf{h}_{\mathrm{b}, k}\right\|^{2}=\left|g_{k}\right|^{2} \sim \operatorname{Exp}(1)$, and the ergodic sum rate becomes $\overline{\mathcal{R}}_{\text {inf }}=\frac{S}{\ln 2} e^{\frac{1}{\rho M}} \Gamma\left(0, \frac{1}{\rho M}\right)$.

Note that as the number of quantization bits decreases to zero, $\overline{\mathcal{R}}_{\text {loss }}(\alpha)$ increases to $\overline{\mathcal{R}}_{\text {inf }}$, which leads $\overline{\mathcal{R}}_{1} \rightarrow 0$. On the other hand, as the number of quantization bits increases to infinity, $\overline{\mathcal{R}}_{\text {loss }}(\alpha)$ decreases to zero, which leads $\overline{\mathcal{R}}_{1} \rightarrow \overline{\mathcal{R}}_{\text {inf. }}$. This complies with intuition.

Now, we focus on the second case where channels have arbitrary AoAs, which leads to the channel leakage effect in the beam domain due to phase offsets. The derived ergodic rate for the second case is shown in Proposition 2.

Proposition 2. When channels have a single path and arbitrary AoAs regardless of the quantized angles of the analog combiner, a lower bound of the ergodic sum rate for $\left|\mathcal{S}_{\mathrm{cd}}\right|=S$ scheduled users with the proposed chordal distance-based scheduling algorithm is approximated as

$$
\begin{aligned}
\overline{\mathcal{R}}_{2}^{l b}=\frac{S}{\ln 2}\left(e^{\frac{1+\rho(1-\alpha)(S-1) M^{2} \mathcal{F}_{2}(M)}{\rho \alpha M+\rho(1-\alpha) M^{2} \mathcal{F}_{1}(M)}} \Gamma\left(0, \frac{1+\rho(1-\alpha)(S-1) M^{2} \mathcal{F}_{2}(M)}{\rho \alpha M+\rho(1-\alpha) M^{2} \mathcal{F}_{1}(M)}\right)\right. \\
\left.-e^{\frac{1+\rho(1-\alpha)(S-1) M^{2} \mathcal{F}_{2}(M)}{\rho(1-\alpha) M^{2} \mathcal{F}_{1}(M)}} \Gamma\left(0, \frac{1+\rho(1-\alpha)(S-1) M^{2} \mathcal{F}_{2}(M)}{\rho(1-\alpha) M^{2} \mathcal{F}_{1}(M)}\right)\right)
\end{aligned}
$$

where $\mathcal{F}_{1}(M)=\int_{0}^{1} F^{4}(\delta, M) d \delta, \mathcal{F}_{2}(M)=\left(\int_{0}^{1} F^{2}(\delta, M) d \delta\right)^{2}$, and $F(\delta, M)$ is the Fejér kernel. Proof. See Appendix B.

Remark 4. The derived ergodic rate expressions in (31) and (34) both converge to $\overline{\mathcal{R}}_{\text {inf }}$ as the number of quantization bits increases:

$$
\overline{\mathcal{R}}_{1}, \overline{\mathcal{R}}_{2}^{l b} \rightarrow \frac{S}{\ln 2} e^{\frac{1}{\rho M}} \Gamma\left(0, \frac{1}{\rho M}\right), \quad \text { as } \alpha \rightarrow 1 .
$$

As the quantization precision increases, the lower bound in (40) becomes an exact expression, and (34) becomes an approximation of the ergodic rate itself rather than its lower bound. Accordingly, it can be inferred from Remark 4 that the two channel scenarios lead to different ergodic rates as a consequence of quantization. In this regard, although a single path channel is considered, Propositions 1 and 2 still convey meaningful information as they not only provide 
closed-form ergodic rates but also specify the channel leakage effect in terms of ergodic rate for low-resolution ADCs. In addition, the single-path channel model is relevant to the case of unmanned aerial vehicle systems [48], which is of interest in upcoming 5G wireless communication systems. In Section V, based on the intuition from Propositions 1 and 2, we show that the channel leakage, indeed, positively affects the ergodic rate in the low-resolution ADC regime, and thus, makes the difference in the ergodic rates of the two channel scenarios.

\section{Simulation Results}

In this section, we numerically evaluate the proposed algorithms, validate the derived ergodic rates, and confirm intuitions in this paper. In simulations, the number of channel paths $L_{k}$ is distributed as $L_{k} \sim \max \left\{\operatorname{Poission}\left(\lambda_{L}\right), 1\right\}$ [42] where $\lambda_{L}$ represents the near average number of channel paths. We consider $M=128 \mathrm{BS}$ antennas and $K=200$ candidate users, and the BS schedules $S=12$ users to serve at each transmission [49], [50]. Without imposing the constraint of $\left\|\mathbf{h}_{\mathrm{b}, k}\right\|=\sqrt{\gamma_{k}}$, the following cases are evaluated through simulation: (1) CSS algorithm, (2) greedy algorithm, (3) chordal distance-based algorithm, (4) mmWave beam aggregation-based scheduling (mBAS) algorithm [28], and (5) SUS algorithm [19]. To provide a reference for a performance lower bound, a random scheduling case is also included. For the CSS and the mBAS algorithms, the BS stores $N_{b}=L_{k}$ indices of dominant elements in the effective channel $\mathbf{h}_{\mathrm{b}, k}$. Parameters such as $\epsilon_{t h}, N_{\mathrm{OL}}$, and $d_{t h}$ are optimally chosen unless mentioned otherwise.

\section{A. Performance Validation}

We first focus on performance validation of the proposed algorithms in sum rate. In Fig. 2, we consider $N=40 \mathrm{RF}$ chains which is about $30 \%$ of the number of antennas $M=128$ and $b=3$ quantization bits. Fig. 2(a) shows the uplink sum rate with respect to the $\operatorname{SNR} \rho$ for $\lambda_{L}=3$. We note that the proposed CSS algorithm achieves the higher sum rate compared to the SUS and mBAS algorithms. In addition, the CSS algorithm attains the sum rate that is comparable to that of the proposed greedy algorithm which achieves the sub-optimal rate by requiring much higher complexity. The sum rate gap between the CSS and the prior algorithms - the SUS and mBAS algorithms - increases as $\rho$ increases because the quantization noise becomes dominant compared to the AWGN in the high SNR regime.

Fig. 2(b) plots simulation results with $\lambda_{L}=8$ average channel paths for $\sum_{k=1}^{S} L_{S}(k)>N$ where the condition in Theorem 1 does not hold. The proposed CSS algorithm achieves a 


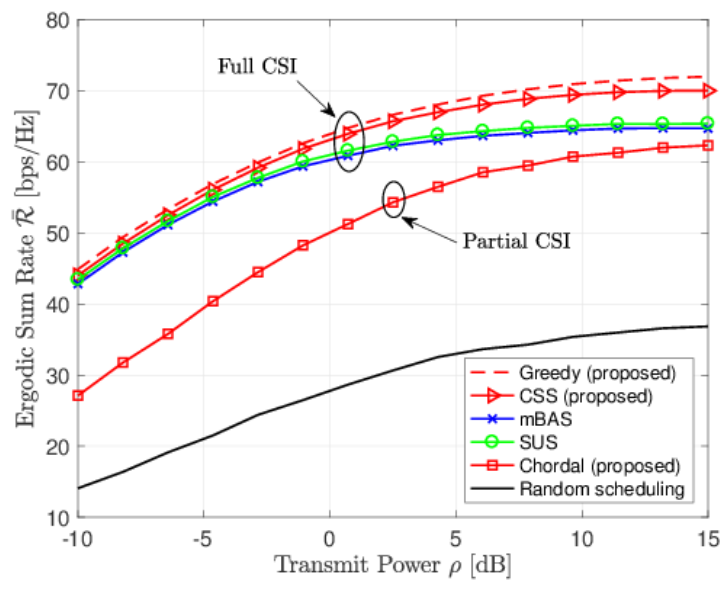

(a) $\lambda_{L}=3$

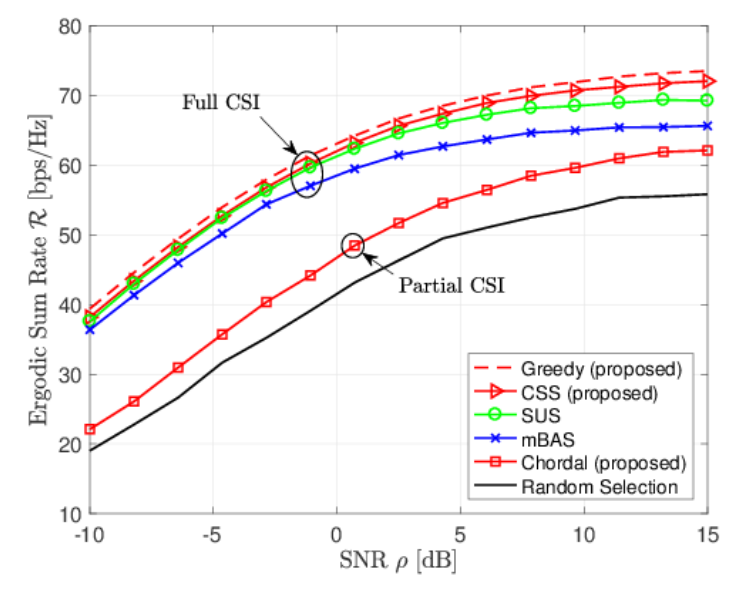

(b) $\lambda_{L}=8$

Fig. 2. Uplink sum rate simulation results for $M=128 \mathrm{BS}$ antennas, $N=40 \mathrm{RF}$ chain, $K=200$ candidate users, $S=12$ scheduled users, and $b=3$ quantization bits with (a) $\lambda_{L}=3$ and (b) $\lambda_{L}=8$ average channel paths,.

higher sum rate than conventional scheduling methods, which shows that although the derived scheduling criteria may not be optimal in a practical system, they can still be effective for mmWave user scheduling as they capture a relationship between the sparse property of mmWave channels and quantization error. In Fig. $2(a)$ and (b), the chordal distance-based algorithm which only exploits the AoA knowledge improves the sum rate compared to random scheduling, closing the gap between the SUS and mBAS algorithms. Therefore, the simulation results validate the sum rate performance of the proposed algorithms.

In Fig. $3(\mathrm{a})$, the sum rate results with respect to the number of RF chains $N$ are presented for $\rho=6 \mathrm{~dB}$. The CCS algorithm shows its sum rate that tightly aligns with that of the greedy algorithm, achieving the higher rate than the SUS and mBAS. In addition, the chordal distancebased algorithm shows a large improvement compared to the random scheduling for the low to medium $N$. As $N$ increases, the effective channels $\mathbf{h}_{\mathrm{b}, k}$ are more likely to be orthogonal to each other for the fixed number of scheduled users, which enhances the performance of random scheduling. In this regard, the sum rates of the SUS and mBAS algorithms show the marginal sum rate increase compared to the random scheduling as $N$ increases, whereas the CSS algorithm still provides the noticeable improvement by mitigating quantization error.

Fig. 3 (b) shows the uplink sum rate with respect to the number of quantization bits $b$. The CSS algorithm also attains the sum rate of the greedy algorithm with lower complexity and 


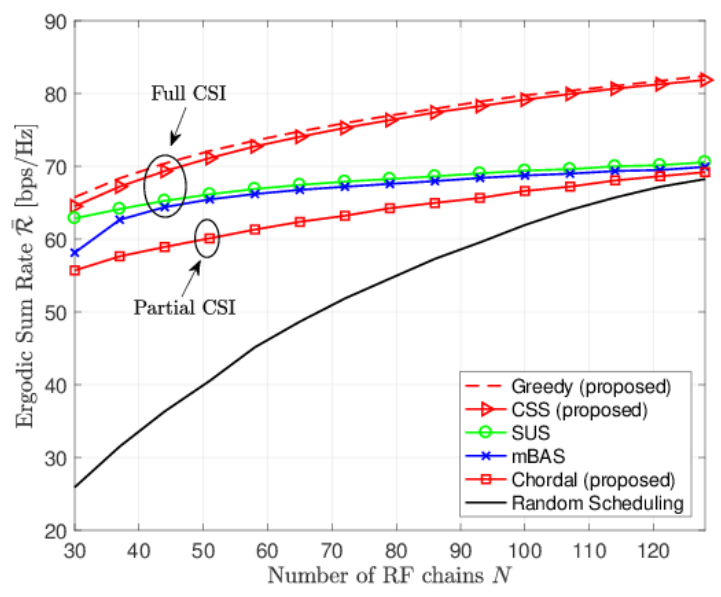

(a)

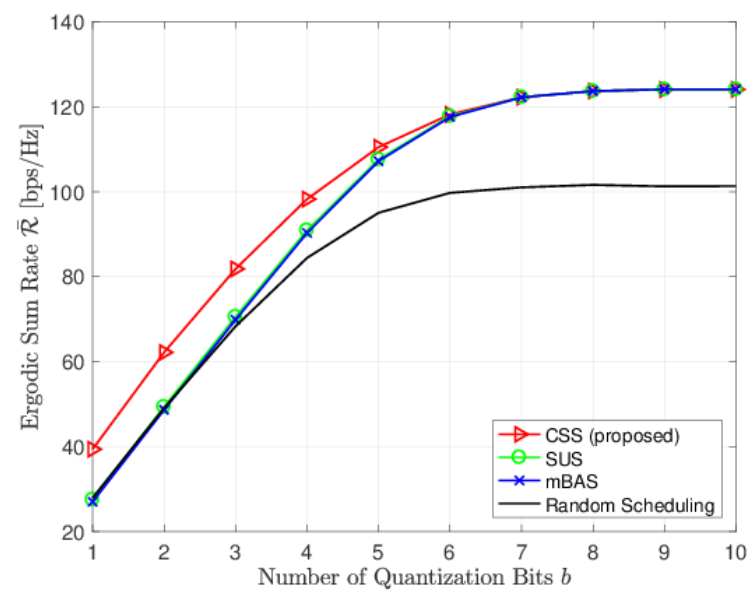

(b)

Fig. 3. Uplink sum rate for $M=128$ antennas, $K=200$ candidate users, $S=12$ scheduled users, $\lambda_{L}=3$ average channel paths, and $\rho=6 \mathrm{~dB}$ SNR with respect to the number of (a) RF chains $N$ with $b=3$ and (b) quantization bits $b$ with $N=128$.

outperforms the SUS and mBAS algorithms. Note that the sum rate of the SUS and mBAS algorithms converges to that of the CSS and greedy algorithms as the number of quantization bits $b$ increases; i.e., quantization error becomes negligible. This convergence corresponds to the fact that the derived criteria is effective under coarse quantization. Thus, in the low-resolution ADC regime, the CSS algorithm provides the noticeable sum rate improvement compared to the other algorithms that ignore quantization error.

\section{B. Analysis Validation}

In this subsection, we validate the performance analysis and intuitions obtained from the analyses. In Fig. 4, we consider $N=128$ and $L_{k}=1, \forall k$. As shown in Fig. 4(a), the derived ergodic rate (31) in Proposition 1 exactly matches the ergodic rate from the simulation. In addition, the lower bound approximation of ergodic rate (34) in Proposition 2 shows a small gap from the ergodic rate of the simulation, validating its analytical accuracy. In this regard, the derived ergodic rates can provide a performance guideline for the hybrid MIMO systems with the proposed chordal distance-based algorithm. From Fig. 4(a), we note that the two different channel scenarios-exact AoA alignment and arbitrary AoAs-show difference in sum rate for the same system configuration, as discussed in Remark 4. In the following simulation results, we numerically examine this phenomenon based on intuitions obtained in this paper. 


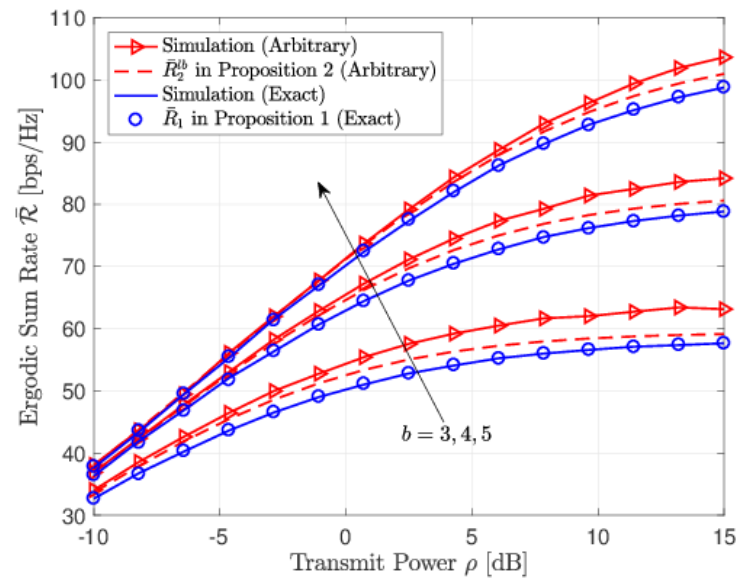

(a)

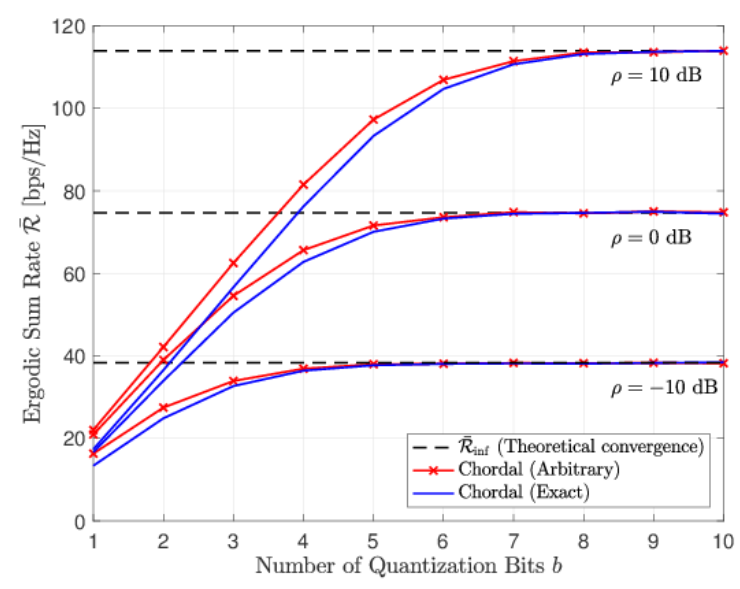

(b)

Fig. 4. (a) The analytical and simulation results for the uplink sum rate of the system with chordal distance-based scheduling, and (b) simulation results for the uplink sum rate of the system with chordal distance-based scheduling for $M=128$ BS antennas, $N=128$ RF chains, $K=200$ candidate users, $S=12$ scheduled users, and $L_{k}=1$ channel path $\forall k$,

We evaluate the sum rate of the chordal distance-based scheduling algorithm with respect to the number of quantization bits $b$ to find the behavior of the sum rate gap between the two channel scenarios: exact AoA alignment and arbitrary AoAs. In Fig. 4(b), it is shown that the uplink sum rates converges to $\overline{\mathcal{R}}_{\text {inf }}=\frac{S}{\ln 2} e^{\frac{1}{\rho M}} \Gamma\left(0, \frac{1}{\rho M}\right)$ as $b$ increases. As discussed in Remark 4, such convergence of the sum rates implies that the two channel scenarios lead to different effects on quantization error. We can also note that the convergence rates are different for different $\rho$. When the SNR is low, the quantization noise is less dominant compared to the AWGN, which results in faster convergence in terms of the number of $b$, and vice versa. Therefore, we can conclude that coarse quantization causes the different sum rates from the channel scenarios.

In Fig. 5, we simulate the sum rates for the two channel scenarios with $N=40, \lambda_{L}=3$, and $b=3$. We note that the sum rate for the arbitrary AoA channel is higher than that for the exact AoA alignment channel in the medium and high SNR regime in which the quantization noise is dominant over the AWGN. The quantization noise variance at the $i$ th ADC is computed as $\mathbb{E}\left[\left|y_{i}-y_{\mathrm{q}, i}\right|^{2}\right]=\frac{\pi \sqrt{3}}{2} \sigma_{i}^{2} 2^{-2 b}[5]$, where $\sigma_{i}^{2}=\mathbb{E}\left[\left|y_{i}\right|^{2}\right]=p_{u}\left\|\left[\mathbf{H}_{\mathrm{b}}\right]_{i,:}\right\|^{2}+1$. Therefore, without the phase offset, most $\sigma_{i}^{2}$ would be large whereas most $\sigma_{i}^{2}$ would be moderate with the phase offsets as the phase offsets spread the channel path gain at certain angles over the entire angles of RF chains. Consequently, the phase offset reduces the overall quantization noise variance and this leads to the performance gain. This corresponds to the results in Theorem 1-(ii), i.e., it is more 


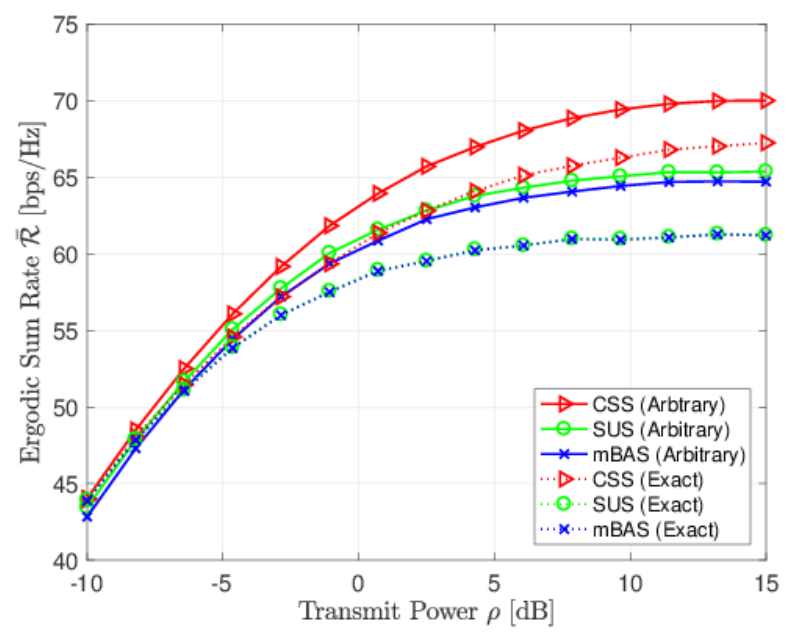

Fig. 5. Uplink sum rate simulation results for $M=128 \mathrm{BS}$ antennas, $N=40 \mathrm{RF}$ chains, $K=200$ candidate users, $S=12$ scheduled users, $\lambda_{L}=3$ average channel paths, and $b=3$ quantization bits.

beneficial to have more spread beamspace gains than to have concentrated beamspace gains.

\section{CONCLUSION}

This paper investigated user scheduling for mmWave hybrid beamforming systems with lowresolution ADCs. We proposed new user scheduling criteria that are effective under coarse quantization. Leveraging the criteria, we developed the user scheduling algorithm which achieves the sub-optimal sum rate with low complexity, outperforming the conventional scheduling algorithms. We further proposed the chordal distance-based scheduling algorithm which only exploits the AoA knowledge of channels. The chordal distance-based scheduling algorithm improved the sum rate compared to the random scheduling case, closing the gap between the full CSIbased conventional scheduling methods as the SNR increases. We also provided the performance analysis for the algorithm in ergodice rate, and the derived rates are the functions of system parameters including quantization bits. We obtained an intuition from the derived rates that channel leakage due to the phase offsets between the arbitrary AoAs and quantized angles of analog combiners offers the sum rate gain by reducing the quantization error compared to the channel without leakage. This intuition challenges the conventional negative understanding towards channel leakage and is validated through simulation. Therefore, for mmWave communications, this paper provides not only new user scheduling algorithms for low-resolution ADC systems, but also new scheduling criteria and intuition for mmWave channels under coarse 
quantization. For potential future work, investigating user scheduling for sub-array based hybrid systems with low-resolution ADCs is desirable to consider more practical receiver architectures.

\section{APPENDIX A}

\section{PROOF OF PROPOSITION 1}

Let the ZF combiner $\mathbf{W}_{\mathrm{zf}}=\mathbf{H}_{\mathrm{b}}\left(\mathcal{S}_{\mathrm{cd}}\right)\left(\mathbf{H}_{\mathrm{b}}\left(\mathcal{S}_{\mathrm{cd}}\right)^{H} \mathbf{H}_{\mathrm{b}}\left(\mathcal{S}_{\mathrm{cd}}\right)\right)^{-1}$. Using the achievable rate (8), the ergodic rate of user $k \in \mathcal{S}_{\mathrm{cd}}$ is defined as

$$
\bar{r}_{k}=\mathbb{E}\left[r_{k}\left(\mathbf{H}_{\mathrm{b}}\left(\mathcal{S}_{\mathrm{cd}}\right)\right)\right]=\mathbb{E}\left[\log _{2}\left(1+\frac{\alpha^{2} \rho}{\mathbf{w}_{\mathrm{zf}, k}^{H} \mathbf{R}_{\mathbf{q q}}\left(\mathbf{H}_{\mathrm{b}}\left(\mathcal{S}_{\mathrm{cd}}\right)\right) \mathbf{w}_{\mathrm{zf}, k}+\alpha^{2}\left\|\mathbf{w}_{\mathrm{zf}, k}\right\|^{2}}\right)\right] .
$$

Based on Remark 3, the algorithm schedules a user $j \in \mathcal{K}_{\mathrm{cd}}$ who provides the smallest value of $\left|\mathbf{a}^{H}\left(\phi_{k}\right) \mathbf{a}\left(\phi_{j}\right)\right|$. Under the assumption of the exact AoA alignment, $\left|\mathbf{a}^{H}\left(\phi_{k}\right) \mathbf{a}\left(\phi_{j}\right)\right|$ is equivalent to zero when $\mathcal{L}_{k} \cap \mathcal{L}_{j}=\emptyset$ for $k \neq j$, i.e., user channels are spatially orthogonal to each other. For the exact AoA alignment scenario with $L=1$, there is only one non-zero element in $\mathbf{h}_{\mathrm{b}, k}$. Accordingly, any scheduled users have to satisfy $\mathcal{L}_{k} \cap \mathcal{L}_{j}=\emptyset$ to avoid rank deficiency of a channel matrix, which can be guaranteed by setting $\left|\mathbf{a}^{H}\left(\phi_{k}\right) \mathbf{a}\left(\phi_{k^{\prime}}\right)\right|<\epsilon_{t h} \ll 1$ in the filtering. Hence, the ZF combiner for user $k \in \mathcal{S}_{\mathrm{cd}}$ becomes $\mathbf{w}_{\mathrm{zf}, k}=\mathbf{h}_{\mathrm{b}, k} /\left\|\mathbf{h}_{\mathrm{b}, k}\right\|^{2}$, and (35) is solved as

$$
\begin{aligned}
& \bar{r}_{k}= \mathbb{E}\left[\log _{2}\left(1+\frac{\alpha \rho\left\|\mathbf{h}_{\mathrm{b}, k}\right\|^{4}}{\rho(1-\alpha) \mathbf{h}_{\mathrm{b}, k}^{H} \operatorname{diag}\left(\mathbf{H}_{\mathrm{b}}\left(\mathcal{S}_{\mathrm{cd}}\right) \mathbf{H}_{\mathrm{b}}\left(\mathcal{S}_{\mathrm{cd}}\right)^{H}\right) \mathbf{h}_{\mathrm{b}, k}+\left\|\mathbf{h}_{\mathrm{b}, k}\right\|^{2}}\right)\right] \\
& \stackrel{(a)}{=} \mathbb{E}\left[\log _{2}\left(1+\frac{\alpha \rho}{(1-\alpha) \rho+1 /\left(M\left|g_{k}\right|^{2}\right)}\right)\right] \\
& \stackrel{(b)}{=} \frac{1}{\ln 2}\left(e^{\frac{1}{\rho M}} \Gamma\left(0, \frac{1}{\rho M}\right)-e^{\frac{1}{\rho(1-\alpha) M}} \Gamma\left(0, \frac{1}{\rho(1-\alpha) M}\right)\right)
\end{aligned}
$$

where $g_{k}$ is the complex gain of the propagation path of user $k$. Here, (a) is from $L=1$ with $\mathcal{L}_{k} \cap \mathcal{L}_{k^{\prime}}=\emptyset$ for $k, k^{\prime} \in \mathcal{S}_{\mathrm{cd}}$, and (b) comes from the fact that $\left|g_{k}\right|^{2}$ is an exponential random variable with the rate parameter $\lambda=1,\left|g_{k}\right|^{2} \sim \operatorname{Exp}(1)$. Due to the randomness of $g_{k}$, the ergodic rate of each user is equal, which leads to (31). This completes the proof.

\section{APPENDIX B}

\section{PROOF OF PROPOSITION 2}

To find a lower bound of the ergodic sum rate achieved by the proposed algorithm, we consider the random scheduling method and find its ergodic sum rate for the lower bound. Since we focus on a large antenna array system at the BS, the array response vectors of the scheduled users are 
almost orthogonal with large $M[52]$, and thus we adopt $\mathbf{w}_{\mathrm{zf}, k} \approx \frac{\mathbf{A}^{H} \mathbf{h}_{k}}{\left\|\mathbf{h}_{k}\right\|^{2}}$. Then, the ergodic rate of the scheduled user $k$ can be approximated as

$$
\begin{aligned}
& \bar{r}_{k}=\mathbb{E}\left[\log _{2}\left(1+\frac{\alpha^{2} \rho}{\mathbf{w}_{\mathrm{zf}, k}^{H} \mathbf{R}_{\mathbf{q q}}\left(\mathbf{H}_{\mathrm{b}}\left(\mathcal{S}_{\mathrm{cd}}\right)\right) \mathbf{w}_{\mathrm{zf}, k}+\alpha^{2}\left\|\mathbf{w}_{\mathrm{zf}, k}\right\|^{2}}\right)\right] \\
& \stackrel{(a)}{\approx} \mathbb{E}\left[\log _{2}\left(1+\frac{\alpha \rho\left\|\mathbf{h}_{k}\right\|^{4}}{(1-\alpha)\left(\mathbf{A}^{H} \mathbf{h}_{k}\right)^{H} \operatorname{diag}\left(\rho \mathbf{A}^{H} \mathbf{H}\left(\mathcal{S}_{\mathrm{cd}}\right) \mathbf{H}^{H}\left(\mathcal{S}_{\mathrm{cd}}\right) \mathbf{A}\right) \mathbf{A}^{H} \mathbf{h}_{k}+\left\|\mathbf{h}_{k}\right\|^{2}}\right)\right],
\end{aligned}
$$

where (a) comes from $\mathbf{w}_{\mathrm{zf}, k} \approx \frac{\mathbf{A}^{H} \mathbf{h}_{k}}{\left\|\mathbf{h}_{k}\right\|^{2}}$. Without loss of generality, let $\mathcal{S}_{\mathrm{cd}}=\{1,2, \ldots, S\}$. The channel matrix of scheduled users can be represented as $\mathbf{H}\left(\mathcal{S}_{\mathrm{cd}}\right)=\sqrt{M} \mathbf{A}_{u} \mathbf{G}$ where $\mathbf{A}_{u}=$ $\left[\mathbf{a}\left(\varphi_{1}\right), \ldots, \mathbf{a}\left(\varphi_{S}\right)\right]$ and $\mathbf{G}=\operatorname{diag}\left(g_{1}, \ldots, g_{S}\right)$, and 38$)$ becomes

$$
\begin{aligned}
& \mathbb{E}\left[\log _{2}\left(1+\frac{M^{2} \alpha \rho\left|g_{k}\right|^{4}}{M^{2} \rho(1-\alpha)\left|g_{k}\right|^{2} \mathbf{a}^{H}\left(\varphi_{k}\right) \mathbf{A} \operatorname{diag}\left(\mathbf{A}^{H} \mathbf{A}_{u} \mathbf{G} \mathbf{G}^{H} \mathbf{A}_{u}^{H} \mathbf{A}\right) \mathbf{A}^{H} \mathbf{a}\left(\varphi_{k}\right)+M\left|g_{k}\right|^{2}}\right)\right] \\
& =\mathbb{E}\left[\log _{2}\left(1+\frac{M \alpha \rho\left|g_{k}\right|^{2}}{M \rho(1-\alpha) \sum_{m, s=1}^{M, S}\left|g_{s}\right|^{2}\left|\mathbf{a}^{H}\left(\vartheta_{m}\right) \mathbf{a}\left(\varphi_{k}\right)\right|^{2}\left|\mathbf{a}^{H}\left(\vartheta_{m}\right) \mathbf{a}\left(\varphi_{s}\right)\right|^{2}+1}\right)\right] \\
& =\mathbb{E}_{g_{k}}\left[\mathbb{E}\left[\log _{2}\left(1+\frac{M \alpha \rho\left|g_{k}\right|^{2}}{M \rho(1-\alpha) \sum_{m, s}\left|g_{s}\right|^{2}\left|\mathbf{a}^{H}\left(\vartheta_{m}\right) \mathbf{a}\left(\varphi_{k}\right)\right|^{2}\left|\mathbf{a}^{H}\left(\vartheta_{m}\right) \mathbf{a}\left(\varphi_{s}\right)\right|^{2}+1}\right) \mid g_{k}\right]\right] .
\end{aligned}
$$

To compute the inner expectation in [39], we can use Lemma 1 in [53] as $g_{k}$ is considered to be a constant given the condition, which makes the signal power and the interference-plus-noise power independent to each other. Let $\Psi_{k}=M \rho(1-\alpha) \sum_{m, s=1}^{M, S}\left|g_{s}\right|^{2}\left|\mathbf{a}^{H}\left(\vartheta_{m}\right) \mathbf{a}\left(\varphi_{k}\right)\right|^{2}\left|\mathbf{a}^{H}\left(\vartheta_{m}\right) \mathbf{a}\left(\varphi_{s}\right)\right|^{2}$, then the inner expectation in (39) becomes

$$
\begin{aligned}
\mathbb{E}\left[\log _{2}\left(1+\frac{M \alpha \rho\left|g_{k}\right|^{2}}{\Psi_{k}+1}\right) \mid g_{k}\right] & \stackrel{(a)}{=} \frac{1}{\ln 2} \int_{0}^{\infty} \frac{e^{-z}}{z}\left(1-e^{-z M \alpha \rho\left|g_{k}\right|^{2}}\right) \mathbb{E}\left[e^{-z \Psi_{k}} \mid g_{k}\right] d z \\
& \geq \frac{(b)}{\ln 2} \int_{0}^{\infty} \frac{e^{-z}}{z}\left(1-e^{-z M \alpha \rho\left|g_{k}\right|^{2}}\right) e^{-z \mathbb{E}\left[\Psi_{k} \mid g_{k}\right]} d z
\end{aligned}
$$

where (a) follows from Lemma 1 in [53] and (b) comes from Jensen's inequality. To compute the expectation in 40 , we rewrite it as

$$
\begin{aligned}
\mathbb{E}\left[\Psi_{k} \mid g_{k}\right]=M \rho(1-\alpha)( & \mathbb{E}\left[\sum_{m=1}^{M}\left|g_{k}\right|^{2}\left|\mathbf{a}^{H}\left(\vartheta_{m}\right) \mathbf{a}\left(\varphi_{k}\right)\right|^{4} \mid g_{k}\right]+ \\
& \left.\mathbb{E}\left[\sum_{m=1}^{M} \sum_{s \neq k}^{S}\left|g_{s}\right|^{2}\left|\mathbf{a}^{H}\left(\vartheta_{m}\right) \mathbf{a}\left(\varphi_{k}\right)\right|^{2}\left|\mathbf{a}^{H}\left(\vartheta_{m}\right) \mathbf{a}\left(\varphi_{s}\right)\right|^{2}\right]\right) .
\end{aligned}
$$


The first expectation term in (41) can be computed as

$$
\mathbb{E}\left[\sum_{m=1}^{M}\left|g_{k}\right|^{2}\left|\mathbf{a}^{H}\left(\vartheta_{m}\right) \mathbf{a}\left(\varphi_{k}\right)\right|^{4} \mid g_{k}\right]=\left|g_{k}\right|^{2} \sum_{m=1}^{M} \mathbb{E}\left[\left|\mathbf{a}^{H}\left(\vartheta_{m}\right) \mathbf{a}\left(\varphi_{k}\right)\right|^{4}\right] \stackrel{(a)}{=}\left|g_{k}\right|^{2} M \int_{0}^{1} F^{4}(\delta ; M) d \delta
$$

where (a) comes from the fact that $\delta_{m, k}:=\vartheta_{m}-\varphi_{k}$ can be regarded as $\delta_{m, k} \stackrel{\text { i.i.d. }}{\sim}$ Unif $[-1,1]$ due to the symmetry of the Fejér kernel of order $M, F(\vartheta ; M)[54]$. Then, with $\mathbb{E}\left[\left|g_{s}\right|^{2}\right]=1$, the second expectation term can be expressed as

$\mathbb{E}\left[\sum_{m=1}^{M} \sum_{s \neq k}^{S}\left|\mathbf{a}^{H}\left(\vartheta_{m}\right) \mathbf{a}\left(\varphi_{k}\right)\right|^{2}\left|\mathbf{a}^{H}\left(\vartheta_{m}\right) \mathbf{a}\left(\varphi_{s}\right)\right|^{2}\right]=\sum_{m=1}^{M} \sum_{s \neq k}^{S} \mathbb{E}\left[\left|\mathbf{a}^{H}\left(\vartheta_{m}\right) \mathbf{a}\left(\varphi_{k}\right)\right|^{2}\right] \mathbb{E}\left[\left|\mathbf{a}^{H}\left(\vartheta_{m}\right) \mathbf{a}\left(\varphi_{s}\right)\right|^{2}\right]$
$=\sum_{m=1}^{M} \sum_{s \neq k}^{S} \mathbb{E}\left[F^{2}\left(\delta_{m, k} ; M\right)\right] \mathbb{E}\left[F^{2}\left(\delta_{m, s} ; M\right)\right]=(S-1) M\left(\int_{0}^{1} F^{2}(\delta ; M) d \delta\right)^{2}$.

Let $c_{1}=M \alpha \rho, c_{2}=M^{2} \rho(1-\alpha) \int_{0}^{1} F^{4}(\delta ; M) d \delta$, and $c_{3}=M^{2} \rho(1-\alpha)(S-1)\left(\int_{0}^{1} F^{2}(\delta ; M) d \delta\right)^{2}$.

From (39), 40), 42), and (43), the ergodic rate $\bar{r}_{k}$ is approximately lower bounded by

$$
\begin{aligned}
\bar{r}_{k} & \approx \mathbb{E}_{g_{k}}\left[\mathbb{E}\left[\log _{2}\left(1+\frac{c_{1}\left|g_{k}\right|^{2}}{\Psi_{k}+1}\right) \mid g_{k}\right]\right] \geq \frac{1}{\ln 2} \mathbb{E}_{g_{k}}\left[\int_{0}^{\infty} \frac{e^{-z}}{z}\left(1-e^{-z c_{1}\left|g_{k}\right|^{2}}\right) e^{-z \mathbb{E}\left[\Psi_{k} \mid g_{k}\right]} d z\right] \\
& =\frac{1}{\ln 2} \int_{0}^{\infty} \frac{e^{-\left(1+c_{3}\right) z}}{z}\left(\mathbb{E}_{g_{k}}\left[e^{-c_{2} z\left|g_{k}\right|^{2}}\right]-\mathbb{E}_{g_{k}}\left[e^{-\left(c_{1}+c_{2}\right) z\left|g_{k}\right|^{2}}\right]\right) d z \\
& \stackrel{(a)}{=} \frac{1}{\ln 2} \int_{0}^{\infty} \frac{e^{-\left(1+c_{3}\right) z}}{z}\left(\frac{1}{1+c_{2} z}-\frac{1}{1+\left(c_{1}+c_{2}\right) z}\right) d z \\
& =\frac{1}{\ln 2}\left(e^{\frac{1+c_{3}}{c_{1}+c_{2}}} \Gamma\left(0, \frac{1+c_{3}}{c_{1}+c_{2}}\right)-e^{\frac{1+c_{3}}{c_{2}}} \Gamma\left(0, \frac{1+c_{3}}{c_{2}}\right)\right)
\end{aligned}
$$

where (a) comes from the Laplace transform of the exponential distribution $\left|g_{k}\right|^{2} \sim \exp (1)$. Without the fading information of channels, the ergodic rate for each user after the user scheduling is equivalent to each other, which results in (34). This completes the proof.

\section{REFERENCES}

[1] Z. Pi and F. Khan, "An introduction to millimeter-wave mobile broadband systems," IEEE Commun. Mag, vol. 49, no. 6, pp. 101-107, Jun. 2011.

[2] J. G. Andrews, S. Buzzi, W. Choi, S. V. Hanly, A. Lozano, A. C. Soong, and J. C. Zhang, "What will 5G be?" IEEE J. Sel. Areas in Commun., vol. 32, no. 6, pp. 1065-1082, Jun. 2014.

[3] T. S. Rappaport, S. Sun, R. Mayzus, H. Zhao, Y. Azar, K. Wang, G. N. Wong, J. K. Schulz, M. Samimi, and F. Gutierrez, "Millimeter wave mobile communications for 5G cellular: It will work!" IEEE Access, vol. 1, pp. 335-349, May 2013.

[4] J. Mo and R. W. Heath, "Capacity analysis of one-bit quantized MIMO systems with transmitter channel state information," IEEE Trans. Signal Process., vol. 63, no. 20, pp. 5498-5512, Jul. 2015. 
[5] O. Orhan, E. Erkip, and S. Rangan, "Low power analog-to-digital conversion in millimeter wave systems: Impact of resolution and bandwidth on performance," in IEEE Info. Theory and App. Work., Feb. 2015, pp. 191-198.

[6] R. W. Heath, N. Gonzalez-Prelcic, S. Rangan, W. Roh, and A. M. Sayeed, "An overview of signal processing techniques for millimeter wave MIMO systems," IEEE J. Sel. Topics in Signal Process., vol. 10, no. 3, pp. 436-453, Feb. 2016.

[7] J. Choi and B. L. Evans, "User Scheduling for Millimeter Wave MIMO Communications with Low-Resolution ADCs," IEEE Int. Conf. on Commun., 2018.

[8] J. Mo, P. Schniter, N. G. Prelcic, and R. W. Heath, "Channel estimation in millimeter wave MIMO systems with one-bit quantization," in Proc. Asilomar Conf. Signals, Systems and Comp., Nov. 2014, pp. 957-961.

[9] J. Choi, J. Mo, and R. W. Heath, "Near maximum-likelihood detector and channel estimator for uplink multiuser massive MIMO systems with one-bit ADCs," IEEE Trans. Commun., vol. 64, no. 5, pp. 2005-2018, Mar. 2016.

[10] Y. Li, C. Tao, G. Seco-Granados, A. Mezghani, A. L. Swindlehurst, and L. Liu, "Channel estimation and performance analysis of one-bit massive MIMO systems," IEEE Trans. on Signal Process., vol. 65, no. 15, pp. 4075-4089, 2017.

[11] C. Rusu, N. González-Prelcic, and R. W. Heath, "Low resolution adaptive compressed sensing for mmWave MIMO receivers," in Asilomar Conf. on Signals, Systems and Computers, 2015, pp. 1138-1143.

[12] C.-K. Wen, C.-J. Wang, S. Jin, K.-K. Wong, and P. Ting, "Bayes-optimal joint channel-and-data estimation for massive MIMO with low-precision ADCs," IEEE Trans. Signal Process., vol. 64, no. 10, pp. 2541-2556, 2016.

[13] S. Wang, Y. Li, and J. Wang, "Multiuser detection for uplink large-scale MIMO under one-bit quantization," in IEEE Int. Conf. Commun., 2014, pp. 4460-4465.

[14] — - "Multiuser Detection in Massive Spatial Modulation MIMO With Low-Resolution ADCs," IEEE Trans. on Wireless Commun., vol. 14, no. 4, pp. 2156-2168, April 2015.

[15] J. Mo, A. Alkhateeb, S. Abu-Surra, and R. W. Heath, "Hybrid architectures with few-bit ADC receivers: Achievable rates and energy-rate tradeoffs," IEEE Trans. on Wireless Commun., vol. 16, no. 4, pp. 2274-2287, 2017.

[16] J. Choi, B. L. Evans, and A. Gatherer, "Resolution-Adaptive Hybrid MIMO Architectures for Millimeter Wave Communications," IEEE Trans. on Signal Process., vol. 65, no. 23, pp. 6201-6216, 2017.

[17] J. Choi, J. Sung, B. L. Evans, and A. Gatherer, "ADC Bit Optimization for Spectrum- and Energy-Efficient Millimeter Wave Communications," in IEEE Global Commun. Conf., Dec 2017, pp. 1-6.

[18] J. Sung, J. Choi, and B. L. Evans, "Narrowband Channel Estimation for Hybrid Beamforming Millimeter Wave Communication Systems with One-Bit Quantization,” in IEEE Int. Conf. Acoustics, Speech, and Signal Process., 2018.

[19] T. Yoo and A. Goldsmith, "On the optimality of multiantenna broadcast scheduling using zero-forcing beamforming," IEEE J. Sel. Areas in Commun., vol. 24, no. 3, pp. 528-541, 2006.

[20] M. Sharif and B. Hassibi, "On the capacity of MIMO broadcast channels with partial side information," IEEE Trans. on Info. Theory, vol. 51, no. 2, pp. 506-522, 2005.

[21] B. Zhou, B. Bai, Y. Li, D. Gu, and Y. Luo, "Chordal distance-based user selection algorithm for the multiuser MIMO downlink with perfect or partial CSIT," in IEEE Int. Conf. Advanced Info. Networking and App., 2011, pp. 77-82.

[22] K. Ko and J. Lee, "Multiuser MIMO user selection based on chordal distance," IEEE Trans. Commun., vol. 60, no. 3, pp. 649-654, 2012.

[23] F. Berggren and R. Jantti, "Multiuser scheduling over rayleigh fading channels," in IEEE Global TeleCommun. Conf., vol. 1, 2003, pp. 158-162.

[24] E. Liu and K. K. Leung, "Expected throughput of the proportional fair scheduling over Rayleigh fading channels," IEEE Commun. Letters, vol. 14, no. 6, 2010.

[25] A. Zafar, M. Shaqfeh, M.-S. Alouini, and H. Alnuweiri, "On multiple users scheduling using superposition coding over Rayleigh fading channels," IEEE Commun. Letters, vol. 17, no. 4, pp. 733-736, 2013. 
[26] R. Rajashekar and L. Hanzo, "User Selection Algorithms for Block Diagonalization Aided Multiuser Downlink mm-Wave Communication," IEEE Access, vol. 5, pp. 5760-5772, 2017.

[27] G. Lee, Y. Sung, and J. Seo, "Randomly-directional beamforming in millimeter-wave multiuser MISO downlink," IEEE Trans. on Wireless Commun., vol. 15, no. 2, pp. 1086-1100, 2016.

[28] G. Lee, Y. Sung, and M. Kountouris, "On the performance of random beamforming in sparse millimeter wave channels," IEEE J. Sel. Topics in Signal Process., vol. 10, no. 3, pp. 560-575, 2016.

[29] R. Rajashekar and L. Hanzo, "Iterative matrix decomposition aided block diagonalization for mm-wave multiuser MIMO systems," IEEE Trans. Wireless Commun., vol. 16, no. 3, pp. 1372-1384, 2017.

[30] A. M. Sayeed, "Deconstructing multiantenna fading channels," IEEE Trans. Signal Process., vol. 50, no. 10, pp. 2563-2579, Nov. 2002

[31] S. Park and R. W. Heath Jr, "Spatial Channel Covariance Estimation for the Hybrid MIMO Architecture: A Compressive Sensing Based Approach,” arXiv preprint arXiv:1711.04207, 2017.

[32] J. Brady, N. Behdad, and A. M. Sayeed, "Beamspace MIMO for millimeter-wave communications: System architecture, modeling, analysis, and measurements," IEEE Trans. on Antennas and Propagation, vol. 61, no. 7, pp. 3814-3827, 2013.

[33] A. Simonsson and A. Furuskar, "Uplink power control in LTE-overview and performance, subtitle: principles and benefits of utilizing rather than compensating for SINR variations,” in IEEE Veh. Technol. Conf., 2008, pp. 1-5.

[34] E. Tejaswi and B. Suresh, "Survey of power control schemes for LTE uplink," Int. J. Computer Science and Inform. Technol., vol. 10, p. 2, 2013.

[35] A. Alkhateeb, O. El Ayach, G. Leus, and R. W. Heath, "Channel estimation and hybrid precoding for millimeter wave cellular systems," IEEE J. Sel. Topics Signal Process., vol. 8, no. 5, pp. 831-846, 2014.

[36] O. El Ayach, S. Rajagopal, S. Abu-Surra, Z. Pi, and R. W. Heath, "Spatially sparse precoding in millimeter wave MIMO systems," IEEE Trans. Wireless Commun., vol. 13, no. 3, pp. 1499-1513, 2014.

[37] A. K. Fletcher, S. Rangan, V. K. Goyal, and K. Ramchandran, "Robust predictive quantization: Analysis and design via convex optimization," IEEE J. Sel. Topics Signal Process., vol. 1, no. 4, pp. 618-632, 2007.

[38] A. Gersho and R. M. Gray, Vector quantization and signal compression. Springer 2012 (originally published 1992).

[39] A. Mezghani and J. A. Nossek, "Capacity lower bound of MIMO channels with output quantization and correlated noise," in IEEE Int. Symposium Info. Theory, 2012.

[40] J. Mo, P. Schniter, and R. W. Heath, "Channel estimation in broadband millimeter wave MIMO systems with few-bit ADCs," IEEE Trans. Signal Process., vol. 66, no. 5, pp. 1141-1154, 2018.

[41] S. Jacobsson, G. Durisi, M. Coldrey, U. Gustavsson, and C. Studer, "Throughput analysis of massive MIMO uplink with low-resolution ADCs," IEEE Trans. Wireless Commun., vol. 16, no. 6, pp. 4038-4051, 2017.

[42] M. R. Akdeniz, Y. Liu, M. K. Samimi, S. Sun, S. Rangan, T. S. Rappaport, and E. Erkip, "Millimeter wave channel modeling and cellular capacity evaluation,” IEEE J. Sel. Areas in Commun., vol. 32, no. 6, pp. 1164-1179, 2014.

[43] P. Viswanath, D. Tse, and R. Laroia, "Opportunistic beamforming using dumb antennas," IEEE Trans. Inf. Theory, vol. 48, no. 6, pp. 1277-1294, 2002.

[44] S. He, J. Wang, Y. Huang, B. Ottersten, and W. Hong, "Codebook-based hybrid precoding for millimeter wave multiuser systems," IEEE Trans. Signal Process, vol. 65, no. 20, pp. 5289-5304, 2017.

[45] J. H. Conway, R. H. Hardin, and N. J. Sloane, "Packing lines, planes, etc.: Packings in Grassmannian spaces," Exper. Math., vol. 5, no. 2, pp. 139-159, 1996.

[46] E. Torkildson, H. Zhang, and U. Madhow, "Channel modeling for millimeter wave MIMO," in IEEE Info. theory and app. workshop, 2010, pp. 1-8. 
[47] A. Alkhateeb, J. Mo, N. Gonzalez-Prelcic, and R. W. Heath, "MIMO precoding and combining solutions for millimeterwave systems," IEEE Commun. Mag., vol. 52, no. 12, pp. 122-131, Dec. 2014.

[48] Y. Zeng, J. Lyu, and R. Zhang, "Cellular-connected UAV: Potentials, challenges and promising technologies," arXiv preprint arXiv:1804.02217v1,, Apr. 2018.

[49] S. Malkowsky, J. Vieira, L. Liu, P. Harris, K. Nieman, N. Kundargi, I. C. Wong, F. Tufvesson, V. Öwall, and O. Edfors, “The World's First Real-Time Testbed for Massive MIMO: Design, Implementation, and Validation,” IEEE Access, vol. 5, pp. 9073-9088, 2017.

[50] J. Vieira, S. Malkowsky, K. Nieman, Z. Miers, N. Kundargi, L. Liu, I. Wong, V. Öwall, O. Edfors, and F. Tufvesson, “A flexible 100-antenna testbed for massive MIMO," in IEEE Globecom Workshops, 2014, pp. 287-293.

[51] X. Yang, W. Lu, N. Wang, K. Nieman, C.-K. Wen, C. Zhang, S. Jin, X. Mu, I. Wong, Y. Huang et al., "Design and implementation of a tdd-based 128-antenna massive MIMO prototype system," China Commun., vol. 14, no. 12, pp. 162-187, 2017.

[52] H. Q. Ngo, E. G. Larsson, and T. L. Marzetta, “Aspects of favorable propagation in massive MIMO,” in European Signal Process. Conf., 2014, pp. 76-80.

[53] K. A. Hamdi, “A useful lemma for capacity analysis of fading interference channels," IEEE Trans. Commun., vol. 58, no. 2,2010 .

[54] R. S. Strichartz, The way of analysis. Jones \& Bartlett Learning, 2000. 